\title{
The MAVRIC-Shift Sequence in SCALE for Radiation Transport and Shielding Calculations with Automated Variance Reduction and Parallel Computing
}

Approved for public release.

Distribution is unlimited.

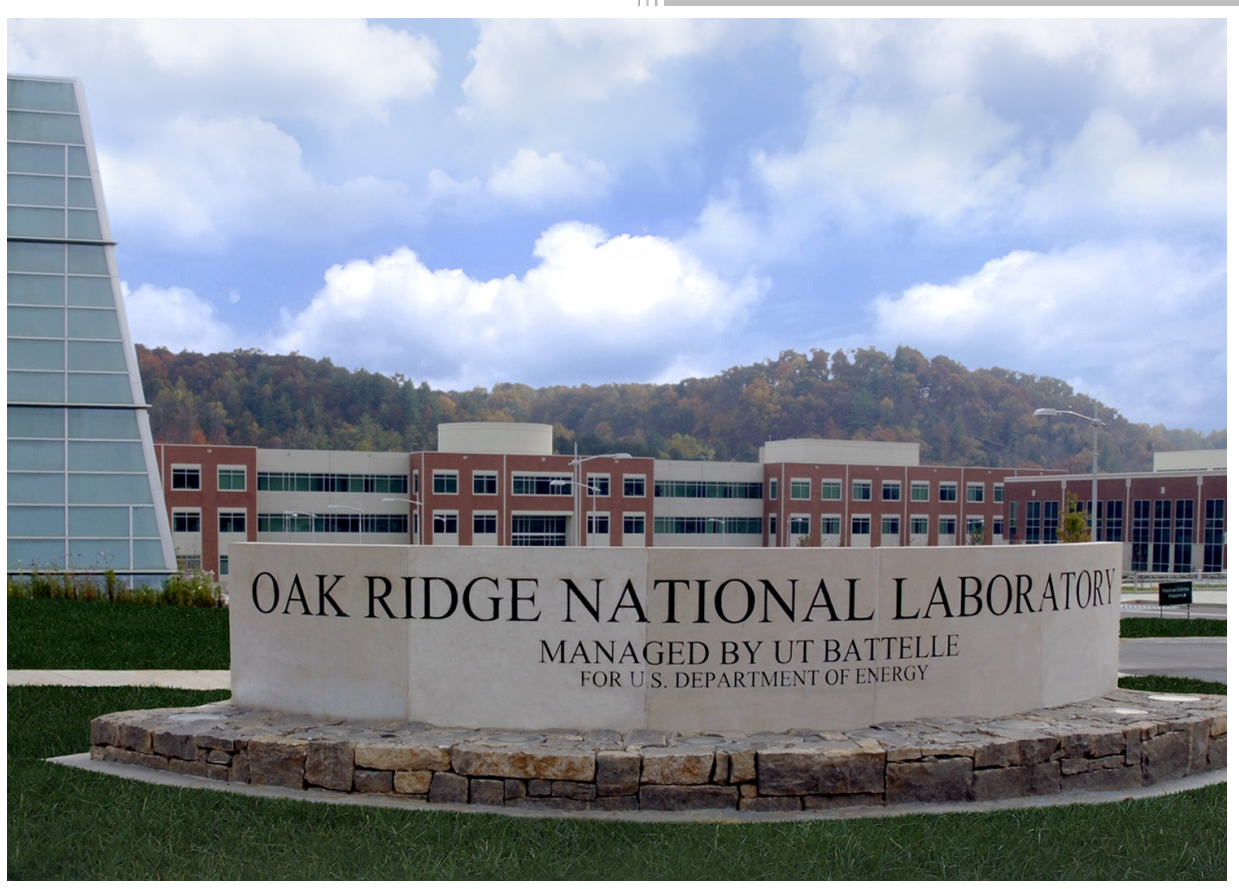

Kaushik Banerjee Cihangir Celik Gregory G. Davidson Thomas M. Evans Bradley T. Rearden William A. Wieselquist

October 28, 2019 


\section{DOCUMENT AVAILABILITY}

Reports produced after January 1, 1996, are generally available free via US Department of Energy (DOE) SciTech Connect.

Website osti.gov

Reports produced before January 1, 1996, may be purchased by members of the public from the following source:

National Technical Information Service

5285 Port Royal Road

Springfield, VA 22161

Telephone 703-605-6000 (1-800-553-6847)

TDD 703-487-4639

Fax 703-605-6900

E-mail info@ntis.gov

Website classic.ntis.gov

Reports are available to DOE employees, DOE contractors, Energy Technology Data Exchange representatives, and International Nuclear Information System representatives from the following source:

Office of Scientific and Technical Information

PO Box 62

Oak Ridge, TN 37831

Telephone 865-576-8401

Fax 865-576-5728

E-mail reports@osti.gov

Website osti.gov/contact

This report was prepared as an account of work sponsored by an agency of the United States Government. Neither the United States Government nor any agency thereof, nor any of their employees, makes any warranty, express or implied, or assumes any legal liability or responsibility for the accuracy, completeness, or usefulness of any information, apparatus, product, or process disclosed, or represents that its use would not infringe privately owned rights. Reference herein to any specific commercial product, process, or service by trade name, trademark, manufacturer, or otherwise, does not necessarily constitute or imply its endorsement, recommendation, or favoring by the United States Government or any agency thereof. The views and opinions of authors expressed herein do not necessarily state or reflect those of the United States Government or any agency thereof. 
ORNL/TM-2019/1358

Reactor and Nuclear Systems Division

\title{
THE MAVRIC-SHIFT SEQUENCE IN SCALE FOR RADIATION TRANSPORT AND SHIELDING CALCULATIONS WITH AUTOMATED VARIANCE REDUCTION AND PARALLEL COMPUTING
}

\author{
Kaushik Banerjee \\ Cihangir Celik \\ Gregory G. Davidson \\ Thomas M. Evans \\ Bradley T. Rearden \\ William A. Wieselquist
}

Date Published: October 28, 2019

Prepared by

OAK RIDGE NATIONAL LABORATORY

Oak Ridge, TN 37831-6283

managed by

UT-Battelle, LLC

for the

US DEPARTMENT OF ENERGY

under contract DE-AC05-00OR22725 


\section{CONTENTS}

Acronyms ....................................... vii

Abstract ......................................1

1. Introduction ................................... 3

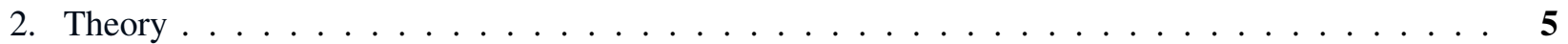

2.1 CADIS and FW-CADIS in the Shift Monte Carlo Code . . . . . . . . . . . . . . . . 5

2.2 Global and Pathlength Weighting . . . . . . . . . . . . . . . . . . 6

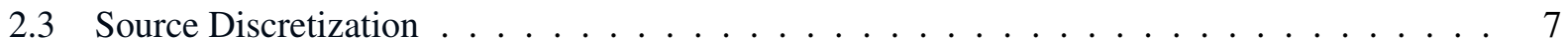

3. Implementation . . . . . . . . . . . . . . . . . . . . . . . . . . . . 11

4. Validation and Verification . . . . . . . . . . . . . . . . . . . . . . 13

4.1 Validation Models . . . . . . . . . . . . . . . . . . . . . . . . 13

4.2 Verification Models . . . . . . . . . . . . . . . . . . . . . . . . . 28

4.3 Additional Shift Verification and Validation . . . . . . . . . . . . . . . . . 46

5. Conclusions and Future Work . . . . . . . . . . . . . . . . . . . . . . . . . . 47 


\section{LIST OF FIGURES}

1 An illustration of Coupling Between Exnihilo and SCALE in the MAVRIC-Shift Sequence. . 11

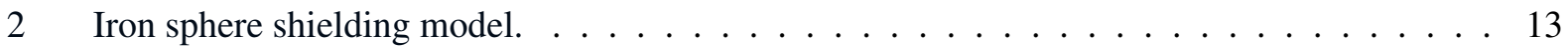

3 Neutron flux comparison for the Czechoslovakian National Research Institute (NRI) mea-

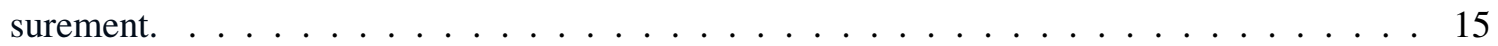

4 Neutron flux comparison for the Skoda measurement. . . . . . . . . . . . . . 16

5 Neutron flux comparison among codes. . . . . . . . . . . . . . . . 17

6 Graphite shielding model. . . . . . . . . . . . . . . . . . . . 18

7 Neutron attenuation rate comparison for the graphite shielding measurement. . . . . . . . 19

8 Neutron attenuation rate comparison among codes. . . . . . . . . . . . . . . 20

9 Dose comparison for the graphite shielding measurement via simulations with implicit cap-

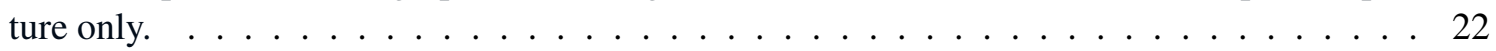

10 Relative uncertainty comparison for the graphite shielding measurement via simulations with implicit capture only. . . . . . . . . . . . . . . . . . . 23

11 Dose comparison for the graphite shielding measurement via CADIS simulations. . . . . . . 24

12 Relative uncertainty comparison for the graphite shielding measurement via CADIS simulations. . . . . . . . . . . . . . . . . . . . . 25

13 FOM comparison for the graphite shielding measurement via simulations with implicit cap-

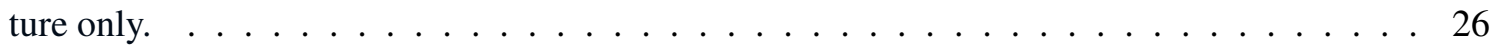

14 FOM comparison for the graphite shielding measurement via CADIS simulations. . . . . . 27

15 Simplified AOS-100 cask model. . . . . . . . . . . . . . . . . . . . . . 28

16 Axial dose rate map (in mrem/h) for the AOS-100 cask model with MAVRIC-Monaco v6.3b6 (a) and MAVRIC-Shift v6.3b6 (b) with their relative uncertainties (c) and (d). . . . . 29

17 Radial dose rate map (in mrem/h) for the AOS-100 cask model with MAVRIC-Monaco v6.3b6 (a) and MAVRIC-Shift v6.3b6 (b) with their relative uncertainties (c) and (d). . . . . 30

18 Simplified TN-24P cask model. . . . . . . . . . . . . . . . . . . . 31

19 Simplified MPC-32 canister model. . . . . . . . . . . . . . . . . . . . . 33

20 Axial neutron dose rate map (in $\mathrm{mrem} / \mathrm{h}$ ) for the MPC-32 cask model with MAVRICMonaco v6.3b6 (a) and MAVRIC-Shift v6.3b6 (b) with their relative uncertainties (c) and (d) . . . . . . . . . . . . . . . . . . . . . . .

21 Radial neutron dose rate map (in mrem/h) for the MPC-32 cask model with MAVRICMonaco v6.3b6 (a) and MAVRIC-Shift v6.3b6 (b) with their relative uncertainties (c) and

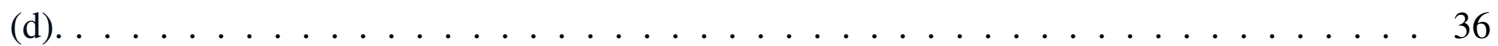

22 Axial gamma dose rate map (in $\mathrm{mrem} / \mathrm{h}$ ) for the MPC-32 cask model with MAVRICMonaco v6.3b6 (a) and MAVRIC-Shift v6.3b6 (b) with their relative uncertainties (c) and (d). . . . . . . . . . . . . . . . . . . . . . .

23 Radial gamma dose rate map (in mrem/h) for the MPC-32 cask model with MAVRICMonaco v6.3b6 (a) and MAVRIC-Shift v6.3b6 (b) with their relative uncertainties (c) and

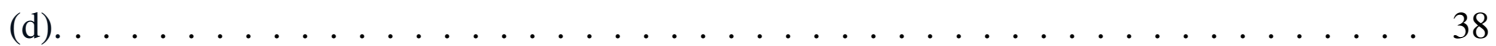

24 Axial total dose rate map (in mrem/h) for the MPC-32 cask model with MAVRIC-Monaco v6.3b6 (a) and MAVRIC-Shift v6.3b6 (b) with their relative uncertainties (c) and (d). . . . . 39

25 Radial total dose rate map (in mrem/h) for the MPC-32 cask model with MAVRIC-Monaco v6.3b6 (a) and MAVRIC-Shift v6.3b6 (b) with their relative uncertainties (c) and (d). . . . . 40

26 Simplified canister model with detailed source definitions. . . . . . . . . . . . . . 41

27 Neutron flux comparison between codes. . . . . . . . . . . . . . . . . . . . 42

28 Simplified ISFSI model. . . . . . . . . . . . . . . . . . . . . . . 43 
29 Axial gamma dose rate map (in rem/h) for the ISFSI model with MAVRIC-Monaco v6.3b6 (a) and MAVRIC-Shift v6.3b6 (b) with their relative uncertainties (c) and (d). . . . . . . . . 44 30 Radial gamma dose rate map (in rem/h) for the ISFSI model with MAVRIC-Monaco v6.3b6 (a) and MAVRIC-Shift v6.3b6 (b) with their relative uncertainties (c) and (d). . . . . . . . . 45 


\section{LIST OF TABLES}

1 Adjoint source options in MAVRIC-Shift. . . . . . . . . . . . . . . . 6

2 Spatial source distributions supported by Shift and the type of discretization method used. $\quad 8$

3 Timing results for single processor iron sphere shielding simulations. . . . . . . . . . . . . 14

4 Timing results for single processor graphite shielding simulations with no shield. . . . . . . 19

5 Timing results for single processor graphite shielding simulations with $20 \mathrm{~cm}$ graphite shield. 20

6 Timing results for serial and parallel processor graphite shielding simulations with $20 \mathrm{~cm}$ graphite shield and $100 \times N\left(10^{7}\right)$ particles using CADIS. . . . . . . . . . . . . . 21

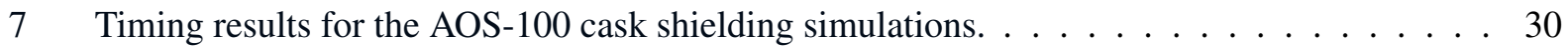

8 Calculated dose values for the TN-24P cask shielding simulations. . . . . . . . . . . 32

9 Timing results for the TN-24P cask shielding simulations. . . . . . . . . . . . . . 32

10 Timing results for the MPC-32 canister shielding simulations. . . . . . . . . . . . . 34

11 Timing results for the canister shielding simulations. . . . . . . . . . . . 42

12 Timing results for the ISFSI simulations. . . . . . . . . . . . . . 44 


\section{ACRONYMS}

$\begin{array}{ll}\text { ADVANTG } & \text { Automated Variance Reduction Generator } \\ \text { ANSI } & \text { American National Standards Institute } \\ \text { AOS } & \text { Alpha Omega Services Inc. } \\ \text { BWR } & \text { boiling water reactor } \\ \text { CADIS } & \text { consistent adjoint-driven importance sampling } \\ \text { ENDF } & \text { Evaluated Nuclear Data File } \\ \text { FOM } & \text { figure-of-merit } \\ \text { FW-CADIS } & \text { forward-weighted consistent adjoint-driven importance sampling } \\ \text { HPC } & \text { high performance computing } \\ \text { ISFSI } & \text { Independent Spent Fuel Storage Installation } \\ \text { MAVRIC } & \text { Monaco with Automated Variance Reduction using Importance Calculations } \\ \text { MC } & \text { Monte Carlo } \\ \text { NRC } & \text { Nuclear Regulatory Commission } \\ \text { NRI } & \text { Czechoslovakian National Research Institute } \\ \text { PWR } & \text { pressurized water reactor } \\ \text { SNF } & \text { spent nuclear fuel } \\ \text { URR } & \text { unresolved resonance region }\end{array}$




\begin{abstract}
A new MAVRIC-Shift sequence has been implemented and tested within the SCALE Code System to provide parallel computing capabilities for radiation transport and shielding analysis with automated variance reduction. This new sequence provides the most widely used capabilities of the MAVRIC sequence and also features the highly scalable Shift Monte Carlo solver, thus enabling faster time-to-solution when run on multiple cores.

This report provides a summary of the theory of variance reduction approaches in MAVRIC and the integration of Shift into the MAVRIC sequence of SCALE. Similarities and differences in methodologies between the traditional MAVRIC sequence and the new MAVRIC-Shift sequence are discussed. Validation cases are presented with models of radiation shielding experiments and verification is provided with a series of practical application cases of interest to the US Nuclear Regulatory Commission, the sponsors of this work, including spent nuclear fuel transportation and storage systems.
\end{abstract}




\section{INTRODUCTION}

This report presents a modernized SCALE MAVRIC (Monaco with Automated Variance Reduction Using Importance Calculations) fixed source radiation transport analysis sequence that uses the massively parallel Shift Monte Carlo (MC) transport solver [1]. MC fixed source radiation transport calculations are primarily used for developing shielding design for various systems containing radioactive sources including nuclear reactors, spent nuclear fuel (SNF) storage, transportation, and disposal systems, and medical facilities. MC fixed source radiation transport calculations are also used to evaluate energy deposition in various structural/shielding materials such as the reactor vessel to predict radiation-related structural damage.

Radiation shielding analysis is primarily performed to evaluate dose or dose rates. The dose rate is used to determine the effectiveness of a system design from a radiation protection perspective by showing that it complies with the applicable federal regulations and that the design supports the principle of maintaining the dose at a level that is as low as reasonably achievable (ALARA). Therefore, radiation shielding analysis is an integral part of a new system design development and is necessary to support the licensing process of that system. MC is used because of its ability to accurately represent complex geometry and physics. However, MC radiation transport analyses of deep penetrating problems are computationally expensive and require variance reduction techniques to obtain meaningful results in a reasonable time frame. The MAVRIC sequence within SCALE implements the consistent adjoint-driven importance sampling (CADIS) and forward-weighted consistent adjoint-driven importance sampling (FW-CADIS) methodologies to provide automated variance reduction, which is reviewed in Section 2 of this report.

The most recent production release of MAVRIC in SCALE 6.2.3 [2] uses the Denovo deterministic code [3] for forward and adjoint calculations and the Monaco MC code for forward radiation transport evaluation. Monaco is a serial MC code, and while Denovo is a parallel three-dimensional $\mathrm{S}_{N}$ code, the MAVRIC sequence up through SCALE 6.2.3 does not allow for parallel execution of Denovo. Therefore, the current production MAVRIC sequence is single processor-based and cannot take advantage of modern computing platforms. Moreover, shielding analyses have become more complex to support (1) dose analysis of large facilities containing thousands of SNF casks, (2) current reactor fleet life extension (e.g., detailed reactor vessel fluence and ex-core analysis to gauge any radiation damage), and (3) efficient use of various nuclear system designs to include maximum utilization of SNF cask capacity. Although MAVRIC provides a state-of-the-art variance reduction methodology, high-fidelity simulation of highly detailed or spatially large shielding problems using a serial MAVRIC calculation is becoming onerous. The MAVRIC-Shift sequence combines two approaches for accelerating simulations: state-of-the-art variance reduction with parallel Denovo deterministic calculations and parallel MC simulation using the massively parallel Shift MC code. The MAVRIC-Shift sequence is designed to support existing MAVRIC-Monaco input structures. With the few exceptions noted in this report, users should be able to run their existing MAVRIC input(s) using the MAVRIC-Shift sequence with significant runtime improvement on parallel computing hardware.

With parallel computing, the MAVRIC-Shift sequence will significantly improve the computational performance of many shielding analysis problems of interest. An immediate application of the MAVRICShift sequence is a canister-specific as-loaded shielding analysis of SNF storage and transportation systems within Used Nuclear Fuel - Storage, Transportation \& Disposal Analysis Resource and Data System (UNFST\&DARDS) [4]. UNF-ST\&DARDS provides a comprehensive SNF database and a platform for various automated analyses using the SCALE code system. A unique feature of UNF-ST\&DARDS is as-loaded

safety analyses (e.g., criticality, shielding), which is implemented by modeling the actual content of the loaded canisters. As-loaded analysis provides uncredited safety margins with many potential applications. One of the shielding applications of uncredited safety margin is to support large-scale transportation after 
extended storage of SNF. Detailed as-loaded shielding analysis of each canister can provide a more realistic estimate of dose to the general public during transportation that can be used for nationwide transportation planning, thus minimizing transportation risk. However, as-loaded shielding analysis using the SCALE MAVRIC-Monaco serial sequence of each loaded cask in the United States is computationally prohibitive because detailed as-loaded shielding analysis of an SNF cask using the single processor MAVRIC-Monaco sequence takes 3-5 days of computing time, and there are more than 3,000 loaded casks in the United States, with an addition of 300 new casks every year. The MAVRIC-Shift sequence will facilitate such a large number of high-fidelity shielding analyses of US SNF cask inventories, resulting in significantly improved large-scale transportation planning.

The MAVRIC-Shift sequence will also enable detailed shielding analysis of SNF interim storage facilities (ISFs) designed to host thousands of casks. The US Nuclear Regulatory Commission (NRC) is currently reviewing two ISF license applications from private industry. ISF shielding analysis is one of a kind, and it requires modeling up to a few kilometers in all directions, including air to account for skyshine. SCALE's original MAVRIC sequence with the Monaco MC code is currently being used to model these ISFs. However, to reduce the computational footprint, dose rate maps surrounding ISFs up to a few kilometers are currently obtained by summing dose rate maps from individual casks. The MAVRIC-Shift sequence is expected to model the whole facility and obtain dose maps surrounding the facility from a single run, significantly reducing computing and postprocessing time. Additional shielding analyses will also be needed for design and work planning in an ISF, including worker dose estimation for each operational step. Simple bounding estimates can be used, but this may result in auxiliary and unnecessary shielding requirements to reduce worker dose, adding to the cost of the entire ISF operation. The MAVRIC-Shift sequence has a faster run time because it takes advantage of modern computing platforms, thus allowing for detailed worker dose assessments with more realistic and flexible planning of various ISF operations in reduced time.

The MAVRIC-Shift sequence also enables high-fidelity fluence evaluations of the reactor vessel and internal components to predict the extent of radiation-related damage over a reactor lifetime. Detailed reactor vessel fluence analysis cannot be performed with reasonable computational resources and time using the current MAVRIC-Monaco sequence. Originally, nuclear plants were licensed to operate for 40 years. NRC has now approved 20-year license extensions to most of the fleet, enabling operation up to 60 years. However, a second round of license renewals to allow operation out to 80 years is expected. Therefore, understanding any reactor vessel damage due to extended exposure to radiation is paramount for the subsequent license renewal to 80 years. MAVRIC-Shift is expected to take a leading role supporting the life extension of the existing reactor fleet.

This report is organized as follows. Section 2 provides a brief discussion on the mathematical theory related to the CADIS and FW-CADIS methods in Shift. SCALE-Shift integration and the implementation of the MAVRIC-Shift sequence are discussed in Section 3. Section 4 presents MAVRIC-Shift validation and verification using several shielding benchmark problems and a UNF-ST\&DARDS cask problem. Finally, Section 5 provides the conclusions and discusses future work. 


\section{THEORY}

The MAVRIC-Shift sequence uses the CADIS and FW-CADIS methods to optimize difficult fixed source MC problems. The transport solver used in this sequence is Shift, a massively parallel MC code. A full description of Shift's capabilities are beyond the scope of this report; however, the code is described in detail in an article by Pandya [1]. Section 2.1 of this report gives an overview of the CADIS and FW-CADIS methods, Section 2.2 describes enhancements made to the MAVRIC-Shift sequence as part of this work to improve the efficiency of mesh (region) tally optimization using FW-CADIS, and Section 2.3 describes algorithmic improvements made to the source discretization methods in MAVRIC-Shift for both CADIS and FW-CADIS.

\subsection{CADIS AND FW-CADIS IN THE SHIFT MONTE CARLO CODE}

The CADIS and FW-CADIS methods are variance reduction methods for MC simulations that use the adjoint function to calculate weight windows and source biasing parameters. In Shift, the adjoint function is approximated using relatively coarse, fast-running deterministic calculations from Denovo [3]. A full description of these techniques is beyond the scope of this report; however, a brief overview is provided below to place the recent improvements to Shift described in Sections 2.2 and 2.3 in context. A general primer on $\mathrm{MC}$ variance reduction, including a detailed discussion of the weight window technique, is given by Booth [5]. Detailed descriptions of the CADIS and FW-CADIS methodologies are provided in the literature [6-8].

In a source-detector context, consider the biased Monte Carlo source,

$$
\hat{q}(\mathbf{r}, E)=\frac{\phi^{\dagger}(\mathbf{r}, E) q(\mathbf{r}, E)}{\left(\phi^{\dagger}, q\right)}=\frac{\phi^{\dagger} q}{R},
$$

where $R$ is the detector response. Using reciprocity, the detector response is defined,

$$
R=\left(\phi^{\dagger}, q\right)=\left(\sigma_{\mathrm{d}}, \phi\right)
$$

where $\sigma_{\mathrm{d}}$ is the probability of response per distance of neutron travel. If source particles are sampled from this biased source, then the overall weight of source particles must be conserved,

$$
w \hat{q}=w_{o} q,
$$

where the unbiased source weight is $w_{o}=1$. This yields a biased source weight corresponding to the biased source of

$$
w=\frac{q}{\hat{q}}=\frac{R}{\phi^{\dagger}} .
$$

This is the target weight in each region of phase-space that is consistent with the source biasing defined in Eq. (1). To keep particles at the target weight, the weight-window technique described by Booth [5] can be used. Each window is centered at $w$ and has a width of $c=w_{\mathrm{u}} / w_{1}$ where the subscripts $u$ and $l$ signify the upper and lower bounds, respectively:

$$
w=\frac{1}{2}\left(w_{1}+w_{\mathrm{u}}\right) .
$$

Generally, the width is an input parameter, and the lower and upper bounds are given by

$$
\begin{aligned}
& w_{1}=\frac{2 w}{1+c}, \\
& w_{\mathrm{u}}=c w_{1} .
\end{aligned}
$$


To calculate the weight windows and biased source, the scalar adjoint flux $\phi^{\dagger}$ is required. This is accomplished by performing a Denovo deterministic calculation on an equivalent problem. Weight windows do not require exact adjoints to be effective, so the objective is to run the coarsest deterministic calculation possible to optimize the total runtime cost.

The CADIS method is very effective at optimizing the MC transport process for single detector regions. However, in many applications, multiple responses across phase-space are required, as when tallying a detailed flux distribution throughout a large region of physical space or simultaneously calculating the response from multiple detector locations. To address this problem, the FW-CADIS method was developed [8]. The FW-CADIS method constructs sources for the deterministic adjoint calculation that are weighted by formulations of the forward flux. This results in an adjoint importance function that incorporates the impact from multiple regions of phase space. In all other aspects, the method is identical to the CADIS method.

\subsection{GLOBAL AND PATHLENGTH WEIGHTING}

In this effort, the two weighting methods employed using the forward flux are referred to as path-length and global weighting based on the terminology used in ADVANTG [9]. For comparison, the original implementation of FW-CADIS in MAVRIC only supports the global weighting method, although it does not use that name [8].

The original implementation of Shift only supported construction of the Denovo adjoint source using the path-length weighting option. While this method works well for optimizing tallies for multiple cells, it is not as effective for optimizing regions or mesh tallies. This is because path-length weighting attempts to preserve the particle density in each region being tallied by including volume integration into the denominator of the adjoint source as shown in Section 2.2.1. While this behavior is desirable in a single tally cell that should have uniform importance across the cell, it can have negative repercussions in mesh tallies. In mesh-based tallies, each mesh cell is optimized individually using this method, which means that small mesh cells will get an inflated adjoint source relative to larger cells. This can lead to preferential tracking toward small mesh cells in the MC simulation. Furthermore, when mesh tallies are used to optimize for an entire problem region using a single-cell mesh tally, the entire region will be assigned the same adjoint source, which can have deleterious effects if there is a strong forward flux gradient across the region.

To remedy these pathologies, Shift has been modified so that cell tallies are optimized using path-length weighting, and mesh tallies are optimized using global weighting. Both methods can be optimized for individual responses, and they can be integrated or binned over energy as described in Sections 2.2.1 and 2.2.2. The new MAVRIC-Shift adjoint source methods are summarized in Table 1.

Table 1. Adjoint source options in MAVRIC-Shift.

\begin{tabular}{|c|c|c|}
\hline Tally type & Default & Comments \\
\hline mesh (region) & global & global and path-length supported \\
\hline cell & path-length & $\begin{array}{l}\text { path-length supported; if global weighting is selected a warning is } \\
\text { emitted and the tally is processed using path-length weighting }\end{array}$ \\
\hline
\end{tabular}

\subsubsection{PATH-LENGTH WEIGHTING}

For a binned response, the adjoint source is given by

$$
q^{\dagger}(\mathbf{r}, E)=\frac{g(\mathbf{r}) \sigma_{\mathrm{d}}(E)}{\sigma_{\mathrm{d}}(E) \int_{V} \phi(\mathbf{r}, E) d V}=\frac{g(\mathbf{r})}{\int_{V} \phi(\mathbf{r}, E) d V} .
$$


For an integrated response, we have

$$
q^{\dagger}(\mathbf{r}, E)=\frac{g(\mathbf{r}) \sigma_{\mathrm{d}}(E)}{\int_{E} \sigma_{\mathrm{d}}(E) \int_{V} \phi(\mathbf{r}, E) d V d E} .
$$

In the above,

$$
g(\mathbf{r})=\left\{\begin{array}{ll}
0 & \mathbf{r} \notin V \\
f(\mathbf{r}) & \mathbf{r} \in V
\end{array},\right.
$$

where $f$ is the volume fraction of the Denovo volume subtended by the tally volume $(V)$, and $f(\mathbf{r}) \in$ $\{0, \ldots, 1\}$. We integrate the flux in each tally cell using

$$
\int_{r \in V} \phi(\mathbf{r}, E) d V=\sum_{i j \in C} \phi_{i j}^{g} f_{i j} V_{i j},
$$

where $C$ is the set of Denovo mesh cells bounded by the tally cells, and the product $f_{i j} V_{i j}$ is the volume of the cell subtended by the tally region. The energy-integrated response is then

$$
\int_{E} \sigma_{\mathrm{d}}(E) \int_{V} \phi(\mathbf{r}, E) d V d E=\sum_{g} \sigma_{\mathrm{d}}^{g} \sum_{i j \in C} \phi_{i j}^{g} f_{i j} V_{i j}
$$

\subsubsection{GLOBAL WEIGHTING}

For a binned response, the adjoint source is given by

$$
q^{\dagger}(\mathbf{r}, E)=\frac{g(\mathbf{r}) \sigma_{\mathrm{d}}(E)}{\sigma_{\mathrm{d}}(E) \phi(\mathbf{r}, E)}=\frac{g(\mathbf{r})}{\phi(\mathbf{r}, E)} .
$$

For an integrated response, we have

$$
q^{\dagger}(\mathbf{r}, E)=\frac{g(\mathbf{r}) \sigma_{\mathrm{d}}(E)}{\int_{E} \sigma_{\mathrm{d}}(E) \phi(\mathbf{r}, E) d E},
$$

where $g(\mathbf{r})$ is defined in Eq. (10). The energy-integrated response is then

$$
\int_{E} \sigma_{\mathrm{d}}(E) \phi(\mathbf{r}, E) d E=\sum_{g} \sigma_{\mathrm{d}}^{g} \phi_{i j}^{g} .
$$

\subsection{SOURCE DISCRETIZATION}

To compute the biased source, the source distribution provided by the user must first be mapped onto the Cartesian weight window grid and group bounds. Therefore, we must calculate

$$
q_{c}^{g}(\hat{\mathbf{\Omega}})=\int_{E^{g+1}}^{E^{g}} \int_{\mathbf{r} \in V_{c}} q(\mathbf{r}, \hat{\mathbf{\Omega}}, E) \mathrm{d} \mathbf{r} \mathrm{d} E,
$$

where $q_{c}^{g}(\hat{\mathbf{\Omega}})$ is the integral of the source over the Cartesian mesh cell $c$ spanning the space $V_{c}$, and over the energy group $g$ bounded by energies $E^{g+1}$ and $E^{g}$. The process of calculating these integrals is referred to as source discretization.

Shift supports both separable (i.e., separable in space and energy) and nonseparable sources. In the case of separable sources, the source may be written as

$$
q(\mathbf{r}, \hat{\mathbf{\Omega}}, E)=Q q_{s}(\mathbf{r}) q_{a}(\hat{\mathbf{\Omega}}) q_{e}(E),
$$


where $Q$ is the source strength in particles per second, and $q_{s}, q_{a}$, and $q_{e}$ are the spatial, angular, and energy distributions of the source, respectively. Substituting Eq. (17) into Eq. (16) we obtain

$$
q_{c}^{g}(\hat{\mathbf{\Omega}})=Q q_{a}(\hat{\mathbf{\Omega}}) \int_{E^{g+1}}^{E^{g}} q_{e}(E) \mathrm{d} E \int_{\mathbf{r} \in V_{c}} q_{s}(\mathbf{r}) \mathrm{d} \mathbf{r} .
$$

In the case of nonseparable sources, the source may be written as

$$
q(\mathbf{r}, \hat{\mathbf{\Omega}}, E)=Q q_{s e}(\mathbf{r}, E) q_{a}(\hat{\mathbf{\Omega}}),
$$

and Eq. (16) then becomes

$$
q_{c}^{g}(\hat{\mathbf{\Omega}})=Q q_{a}(\hat{\mathbf{\Omega}}) \int_{E^{g+1}}^{E^{g}} \int_{\mathbf{r} \in V_{c}} q_{s e}(\mathbf{r}, E) \mathrm{d} \mathbf{r} \mathrm{d} E .
$$

As can be seen in Eq. (18), the spatial and energy discretizations can be computed separately for separable sources. The energy distributions, being one-dimensional integrals along the energy axis, can always be computed analytically, which Shift exploits. Shift supports mono-energetic, line, histogram, and Watt energy distributions.

Some of the spatial distributions can also be discretized analytically. Any distribution that cannot be discretized analytically is instead discretized using point sampling. With point sampling, the distribution is sampled in large statistical batches until the $L_{2}$ norm of the batches distributions converges. Table 2 summarizes the spatial distributions supported by Shift and indicates whether they can currently be analytically discretized.

\section{Table 2. Spatial source distributions supported by Shift and the type of discretization method used.}

\begin{tabular}{ll}
\hline Spatial Distribution & Discretization method \\
\hline Box & Analytic* $^{*}$ \\
Cartesian mesh & Analytic $^{\dagger}$ \\
Point & Analytic \\
Multipoint & Analytic \\
Cylinder & Analytic* \\
Cylinder shell & Analytic* \\
Sphere & Point sampling \\
Spherical shell & Point sampling \\
\hline
\end{tabular}

Analytic* indicates the source will be analytically discretized only if no material or cell filtering is applied.

Analytic $^{\dagger}$ indicates the Cartesian mesh distribution will be analytically discretized only if it is aligned with the weight window grid.

Currently, Shift only supports a single nonseparable source, a Cartesian-histogram distribution. This distribution permits a different source probability in every cell in a Cartesian mesh, as well as in every group. This distribution can currently only be discretized by point sampling.

After the discretized source $q_{c}^{g}(\hat{\boldsymbol{\Omega}})$ has been calculated, the biased source can then be constructed. The biased source is given by the following from Eq. (1):

$$
\hat{q}_{c}^{g}(\hat{\mathbf{\Omega}})=\frac{\phi_{c}^{\dagger g} q_{c}^{g}(\hat{\boldsymbol{\Omega}})}{R}
$$


where $R$ is the source response, per Eq. (2):

$$
R=\sum_{g=1}^{G} \sum_{c} \phi_{c}^{\dagger g} q_{c}^{g} .
$$




\section{IMPLEMENTATION}

The MAVRIC-Shift sequence was designed so that current MAVRIC users can begin using the new MAVRICShift sequence with a very shallow learning curve and with only minimal changes to their pre-existing inputs. Many current MAVRIC inputs will run as-is if the sequence name is simply changed from "mavric" to "mavric-shift." Users running on parallel infrastructures should find that the new MAVRIC-Shift sequence produces essentially the same output as MAVRIC, only faster with parallel computing. This is demonstrated in Section 4.

Shift was originally designed as part of the Exnihilo radiation transport suite, a stand-alone suite of transport solvers, which also includes the Denovo $\mathrm{S}_{N}$ solver, and Omnibus, a user-facing front-end designed especially for high performance computing (HPC) platforms. The MAVRIC-Shift sequence operates by converting SCALE input into an Omnibus input in a manner that is seamless and invisible to users, and then it runs a Shift hybrid sequence to produce results.

The connections between Exnihilo and SCALE, with their subsidiary components, are illustrated in Fig. 1.

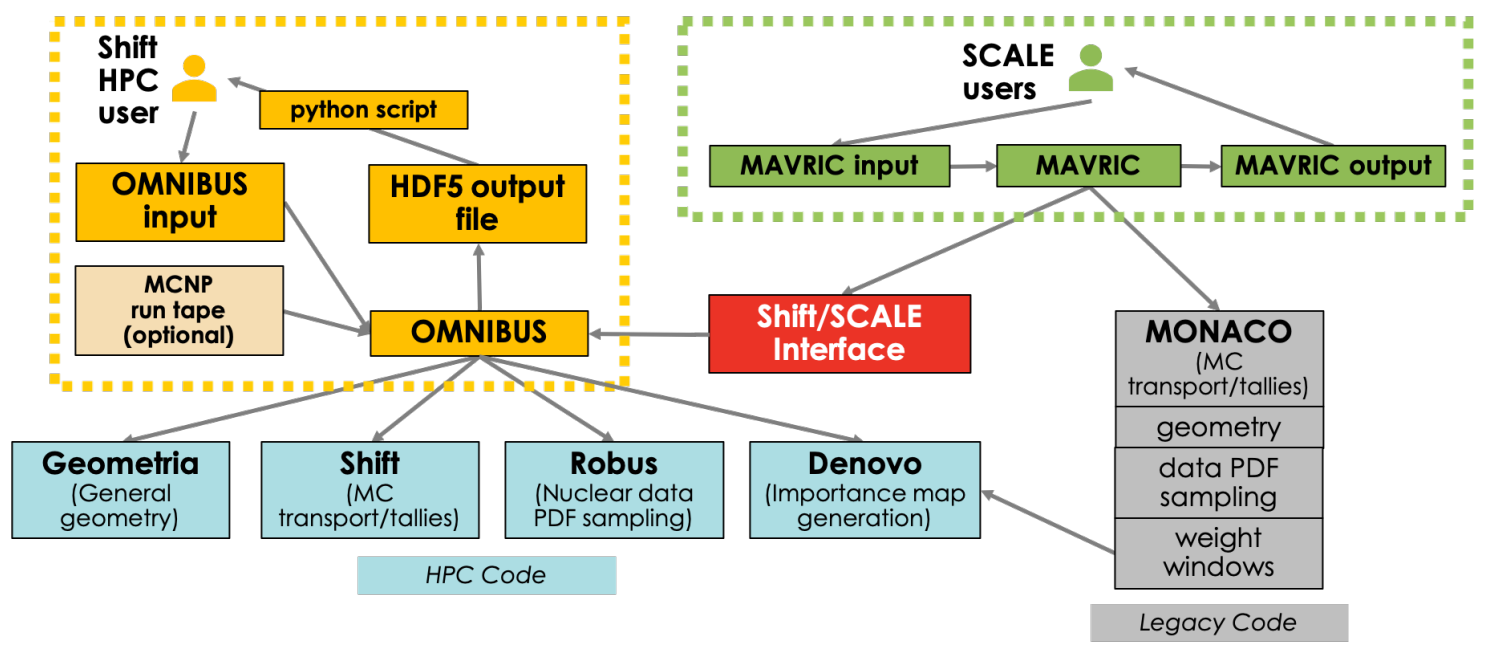

Figure 1. An illustration of Coupling Between Exnihilo and SCALE in the MAVRIC-Shift Sequence.

A SCALE hybrid sequence begins when the user sends input to the MAVRIC sequence driver through the main SCALE executable. If the ordinary MAVRIC sequence has been specified in the input, then that input is used in the regular way: Denovo is run to produce weight windows, and then the input is sent to the Monaco MC code to perform the shielding calculation (shown in grey). However, if the MAVRIC-Shift sequence is specified, then the MAVRIC driver sends the input to the Shift/SCALE Interface (shown in red). The purpose of this interface is to convert SCALE input into a form that the Omnibus driver can understand. After this, a Shift/Denovo hybrid sequence is run.

After the transport solve has finished, the Shift/SCALE interface sends the tally results back to the MAVRIC driver. The MAVRIC driver then interprets these results and compiles them into the standard MAVRIC output format. Because the input and output for the MAVRIC-Shift sequence were designed to mimic the MAVRIC input and output as much as possible, existing MAVRIC users should find using the new MAVRIC-Shift sequence to be straightforward, with faster time to solution.

In the current implementation, some features available in the MAVRIC sequence are not yet supported by the MAVRIC-Shift sequence. 
- The Fulcrum user interface provides numerous visualization capabilities, so some legacy MAVRIC capabilities designed to enable the use of previous SCALE interfaces may no longer be necessarym, such as "make3dmap" and "makeCylMap" for grid geometries and cylindrical geometries and "makeChart" for responses.

- Point detectors are not supported in favor of investing in more effective and general variance reduction methods.

- Importing a fission source mesh (which is generated by KENO and used in the criticality accident alarm system or other fissioning material modeling) as a fixed mesh source is not yet supported, but is planned to be implemented in the future.

- Denovo adjoint and forward fluxes for CADIS and FW-CADIS methods are not saved into individual files for reuse or for investigation of the importance maps; instead, they are internally communicated between Denovo and MAVRIC-Shift. It is anticipated that the ability to save both Denovo adjoint and forward fluxes to an HDF5 file that could be visualized in Fulcrum, as well as reloaded in subsequent calculations, will be enabled in the future.

- Many of the widely used source distribution specifications of MAVRIC are currently implemented in MAVRIC-Shift, including "origensBinaryConcentrationFile," "origensDiscreteGammas," and "wattSpectrum." However, "biasedPDF," "trueCDF," "weight," "importance," and some of the "special" distributions are not supported, so users are advised to supply binned distributions for their models. Similarly, the "mixture" filter is supported, but other source definitions are not yet supported, such as "biasedStrength," "unit" and "region" filters, "rotate" for transforming source shapes, angular distribution keywords ("direction," "dDistributionID," "dScaleDist"), most of the axial spatial distributions (e.g., "xDistributionID," "xScaleDist"), as well as the previously mentioned mesh sources.

- There are also performance-related features that help identify means of reducing the memory requirements or that aid in investigating the model that are available in MAVRIC but are not supported in the MAVRIC-Shift sequence such as "runSampleTest" for distributions, "allowresampling" and "meshsourcefile" in the sources, "allowshortimpmap" in the parameters, and "reduce" in the importance block.

- Time bins for tallies have been used in the MAVRIC sequence but are not supported in the MAVRICShift sequence, mainly due to lack of interest for time-dependent shielding simulations.

Some of the unsupported features are planned for implementation in the future (see Future Work in Section 5). The use of the Fulcrum user interface is highly recommended as it provides automated input checking of inputs against currently implemented features to ensure that all requested parameters are supported with the current version. 


\section{VALIDATION AND VERIFICATION}

A set of validation and verification problems has been used to assess the accuracy and performance of the MAVRIC-Shift sequence. The validation set includes shielding experiments which were measured and published by researchers in the past. The verification suite focuses on cask models which are either representative of or actually used in spent nuclear fuel (SNF) transportation and storage applications by the Nuclear Regulatory Commission (NRC). All validation models were run with the latest stable release (v6.2.3) and the current beta release (v6.3b6) of SCALE. A nuclear data update was implemented in the beta release in which the probability tables were re-normalized to represent neutron cross sections in the unresolved resonance region (URR) [10] more accurately. However, the same Evaluated Nuclear Data File (ENDF) version, ENDF/B-VII.1, has been used for all validation and verification simulations.

\subsection{VALIDATION MODELS}

The objective of the validation models is to confirm the accuracy of the nuclear data and methods used for calculations compared to measurements. Validation models include iron sphere and graphite shielding experiments.

\subsubsection{IRON SPHERE SHIELDING}

A set of neutron transmission experiments through an iron sphere were conducted by Sajo et al. [11] in the early 1990s. The objective of these experiments was to assess neutron inelastic cross sections for iron evaluated in the ENDF/B-VI nuclear data. One configuration from these experiments used in this report was ${ }^{a}{ }^{252} \mathrm{Cf}$ neutron source located at the center of the iron sphere, with measurements of neutron flux at $1 \mathrm{~m}$ away from the source taken by two independent teams, one by NRI and the other by the Skoda Company. The simulation model of the experiment is shown in Fig. 2.

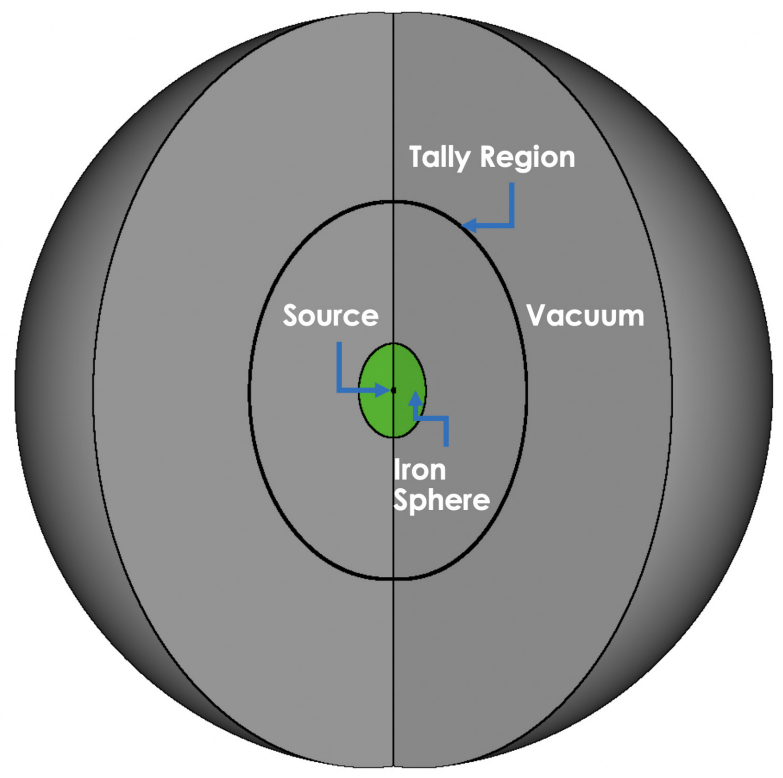

Figure 2. Iron sphere shielding model.

An iron sphere with a radius of $25 \mathrm{~cm}$ in a vacuum was modeled to represent the experiment. A region tally over a spherical shell region, centered at $1 \mathrm{~m}$ apart from the source and $1 \mathrm{~cm}$ thick, was used in the simulation 
to calculate the neutron flux. Skoda and NRI used different equipment and different energy group structures for the flux measurements. Therefore, corresponding group structures were used in the simulations so that separate comparisons can be made for each measurement. Monte Carlo (MC) with only implicit capture and without any other advanced variance reduction methods such as CADIS or FW-CADIS was used in the simulations. Neutron flux spectra are compared in Fig. 3 and Fig. 4. Although there are discrepancies of $>50 \%$ between the measured and calculated spectra below $1 \mathrm{MeV}$ for both measurements, the discrepancies for fast neutron spectra are below $20 \%$, which is typically considered good agreement between calculated and experimental results in shielding applications. Comparisons of the neutron flux spectra between codes shown in Fig. 3 yields very good agreement and verifies the accuracy of MAVRIC-Shift. Timing results for the codes are listed in Table 3, demonstrating that MAVRIC-Shift simulations are performing better than the MAVRIC-Monaco simulations for these problems.

The figure-of-merit (FOM) was used as the metric for comparisons since it represents "quality of the simulations" for a given model and accounts for all the deterministic and $\mathrm{MC}$ components of the simulation. The FOM is a measure of the computational time required to reduce the residual variance in the result. Thus, a higher FOM indicates that a method reduces the variance faster than a result with a lower FOM, indicating a more efficient computational approach. The formula for calculation of the FOM is given by

$$
F O M=\frac{1}{R E^{2} \times T}
$$

where $F O M$ is the figure-of-merit $(1 / \mathrm{min}), R E$ is the relative uncertainty in the tally result, and $T$ is the simulation time in minutes. The FOM values are also included shown in Table 3, and it can be seen that the MAVRIC-Shift results have a higher FOM than MAVRIC.

Table 3. Timing results for single processor iron sphere shielding simulations.

\begin{tabular}{lcccc}
\hline Code & $\begin{array}{c}\text { Number of } \\
\text { processors }\end{array}$ & $\begin{array}{c}\text { CPU time }^{\mathbf{a}} \\
\text { (min) }\end{array}$ & $\begin{array}{c}\text { Wall time }^{\mathbf{b}} \\
(\mathbf{m i n})\end{array}$ & $\begin{array}{c}\text { FOM }^{\mathbf{c}} \\
\text { (/min) }\end{array}$ \\
\hline MAVRIC-Monaco [v6.2.3] & 1 & 44 & 44 & $1.41 \mathrm{e} 7$ \\
MAVRIC-Monaco [v6.3b6] & 1 & 32 & 32 & $1.95 \mathrm{e} 7$ \\
MAVRIC-Shift [v6.3b6] & 1 & 17 & 17 & $3.59 \mathrm{e} 7$ \\
\hline
\end{tabular}

\footnotetext{
a Total CPU time by all processors.

b Total execution time.

c Includes both DENOVO and MAVRIC calculations.
} 


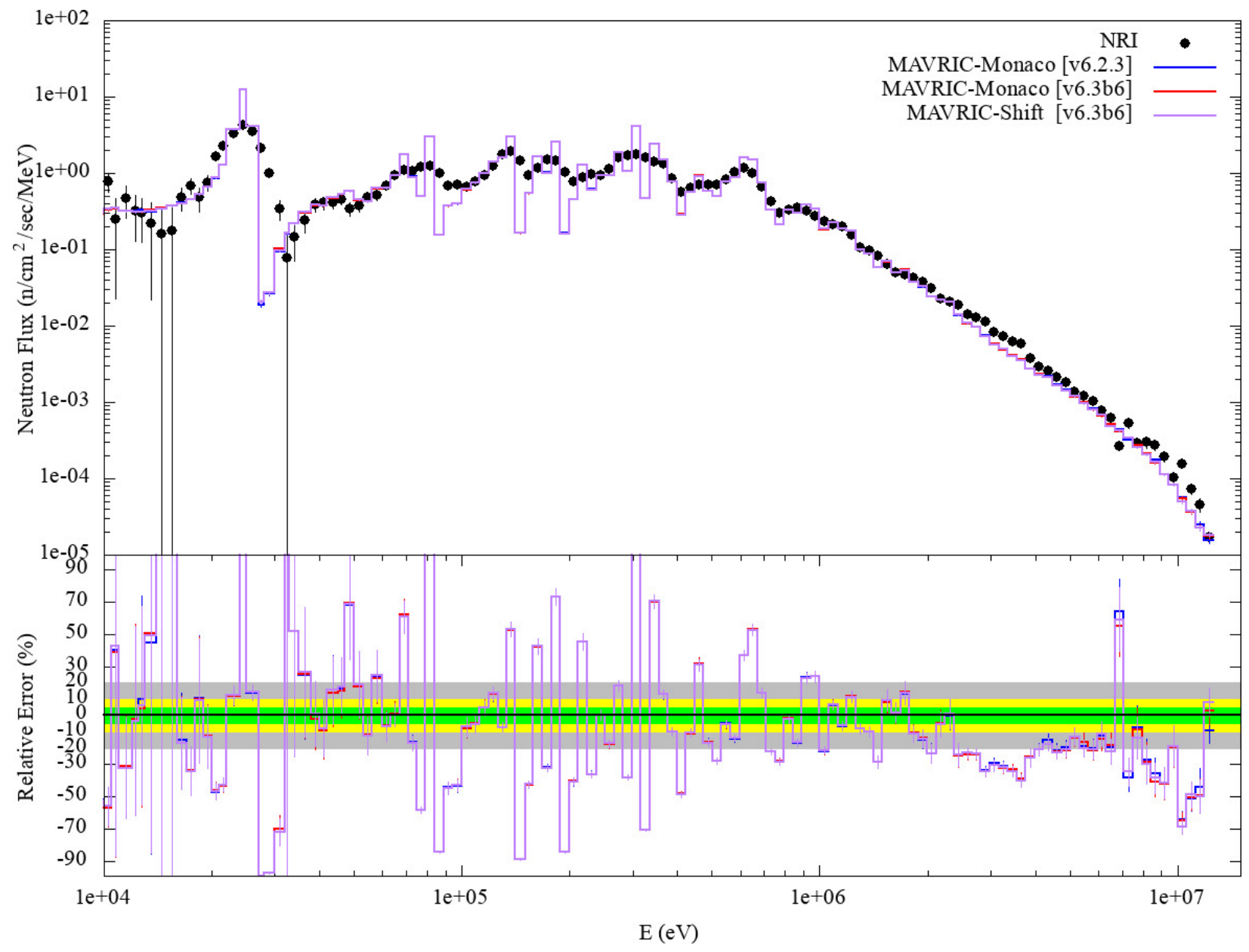

Figure 3. Neutron flux comparison for the NRI measurement. 


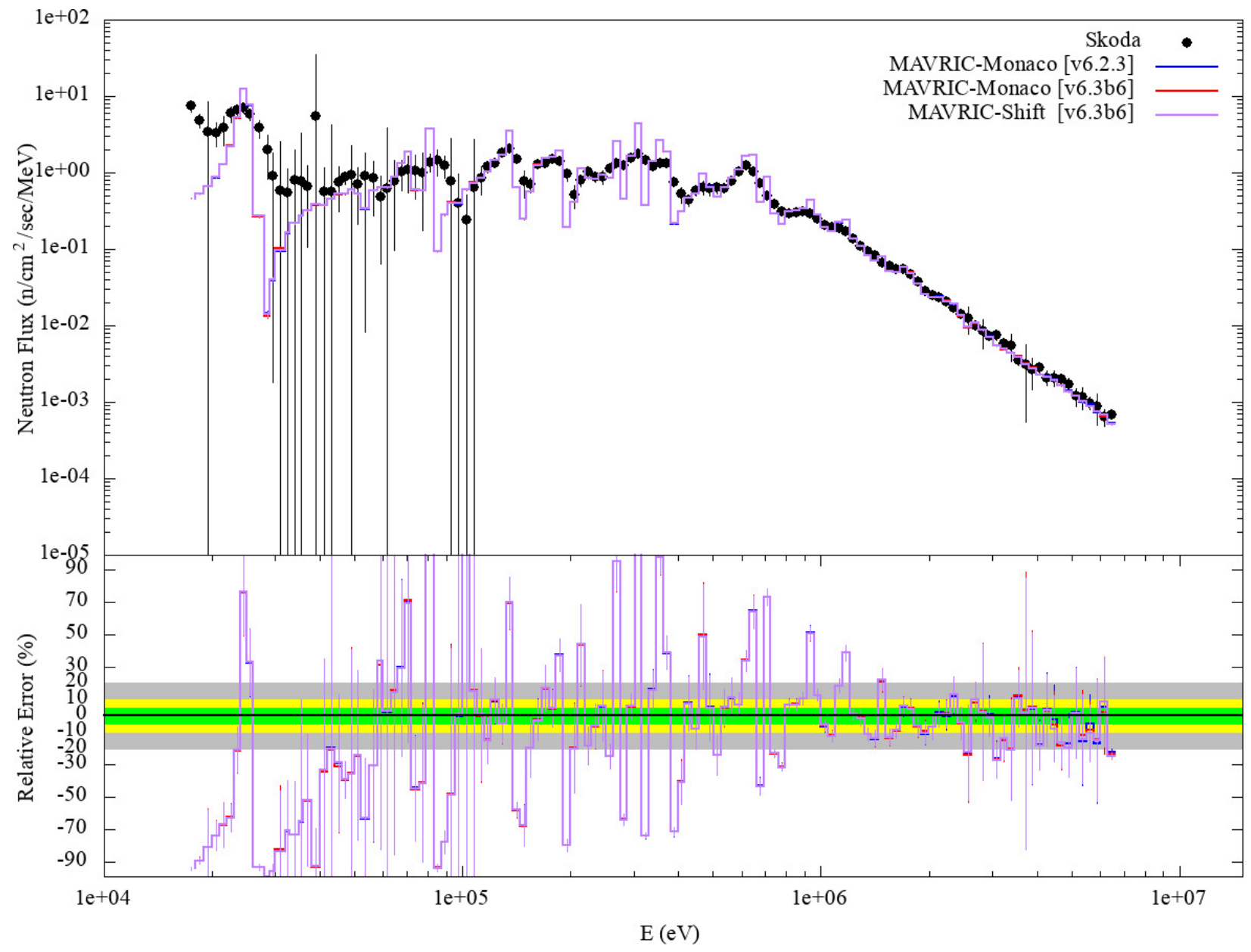

Figure 4. Neutron flux comparison for the Skoda measurement. 


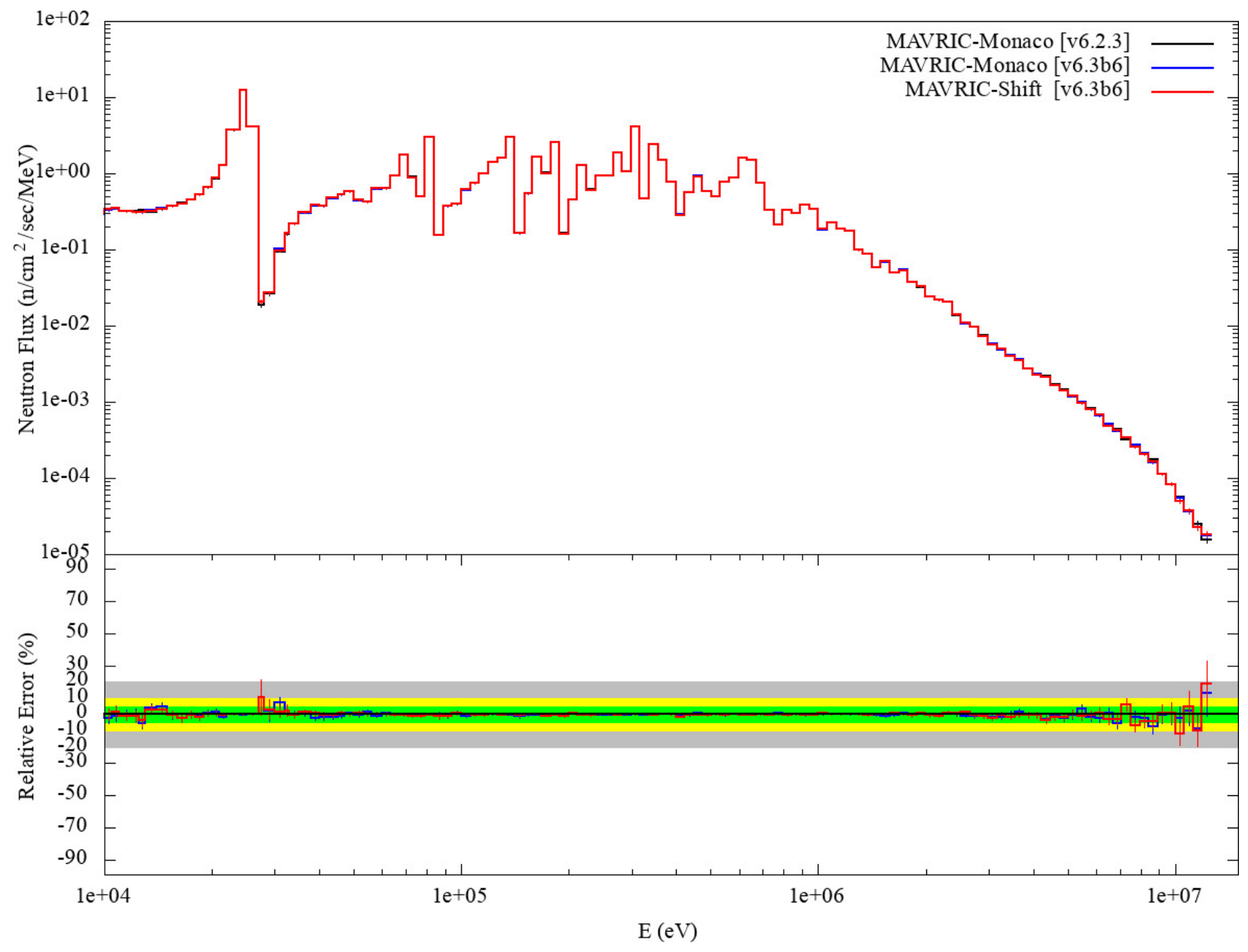

Figure 5. Neutron flux comparison among codes. 


\subsubsection{GRAPHITE SHIELDING}

A set of shielding experiments using various shield blocks was performed by Ueki et al. [12] using either a neutron or a photon source to investigate the effectiveness of common shielding materials. One of these experiments included a ${ }^{252} \mathrm{Cf}$ neutron source located at the center of a paraffin block and shielded by a graphite block. The thickness of the graphite block was increased incrementally using $5 \mathrm{~cm} \times 80 \mathrm{~cm} \times 80 \mathrm{~cm}$ slabs of graphite while keeping the same distance from the source, $90 \mathrm{~cm}$. A neutron detector was placed behind the graphite block and $110 \mathrm{~cm}$ from the source. The neutron attenuation rate of the graphite was reported as the ratio of the measured neutron dose rate behind the graphite shield to the unshielded measured rate. The simulation model of this experiment is shown in Fig. 6.

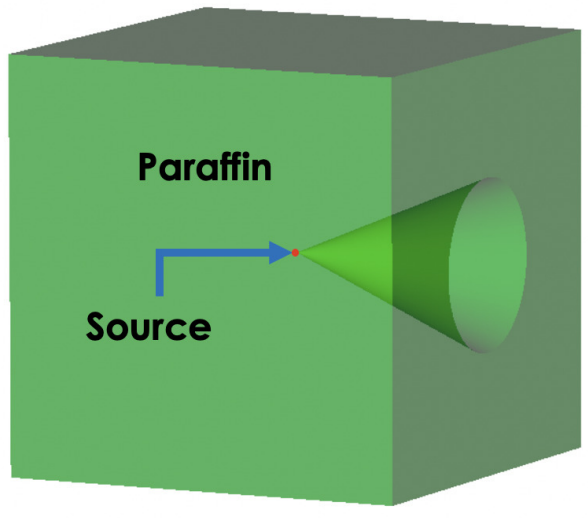

Vacuum

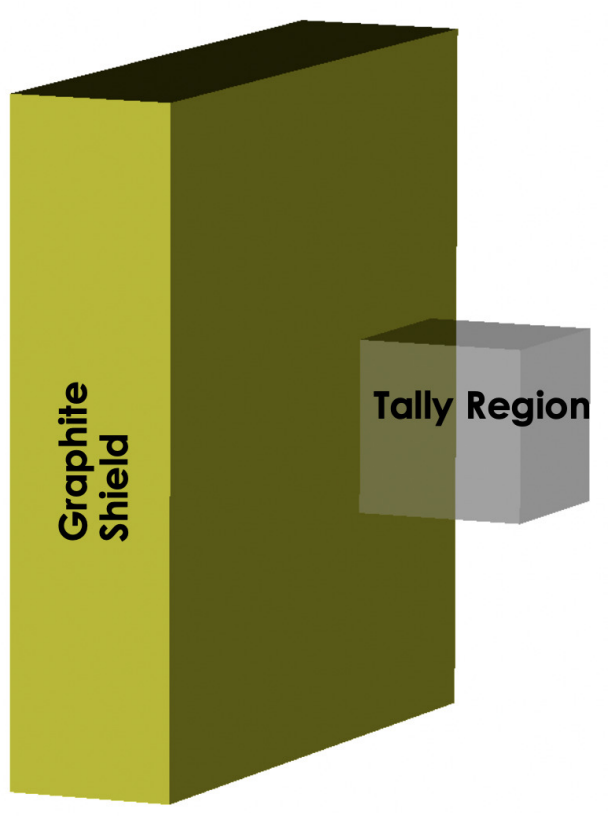

Figure 6. Graphite shielding model.

This experiment was modeled with MAVRIC using the CADIS variance reduction method to calculate the neutron attenuation rate of the graphite. A region tally over a cubical region behind the graphite block, centered at $110 \mathrm{~cm}$ from the source with a $20 \mathrm{~cm}$ side length, was used in the simulation to calculate the neutron dose rate using the American National Standards Institute (ANSI) standard (1977) neutron flux-todose-rate factors [13]. Comparison of the measured and calculated neutron attenuation rates is shown in Fig. 7. The good agreement among the measurement and calculations indicates the accuracy of data and methods in the simulations. Comparisons between the calculated results is shown in Fig. 8 and confirms the accurate implementation of the CADIS method in MAVRIC-Shift. Timing results are listed in Table 4 for the model without the graphite block (bare) and Table 5 for the model with the graphite block (shielded). Although the performance of the codes are comparable in the bare problem, for the shielded problem, the MAVRIC-Shift simulations took longer than the MAVRIC-Monaco simulations, but resulted in a higher FOM value because of the more rigorous source biasing and discretization methods used in MAVRIC-Shift. 


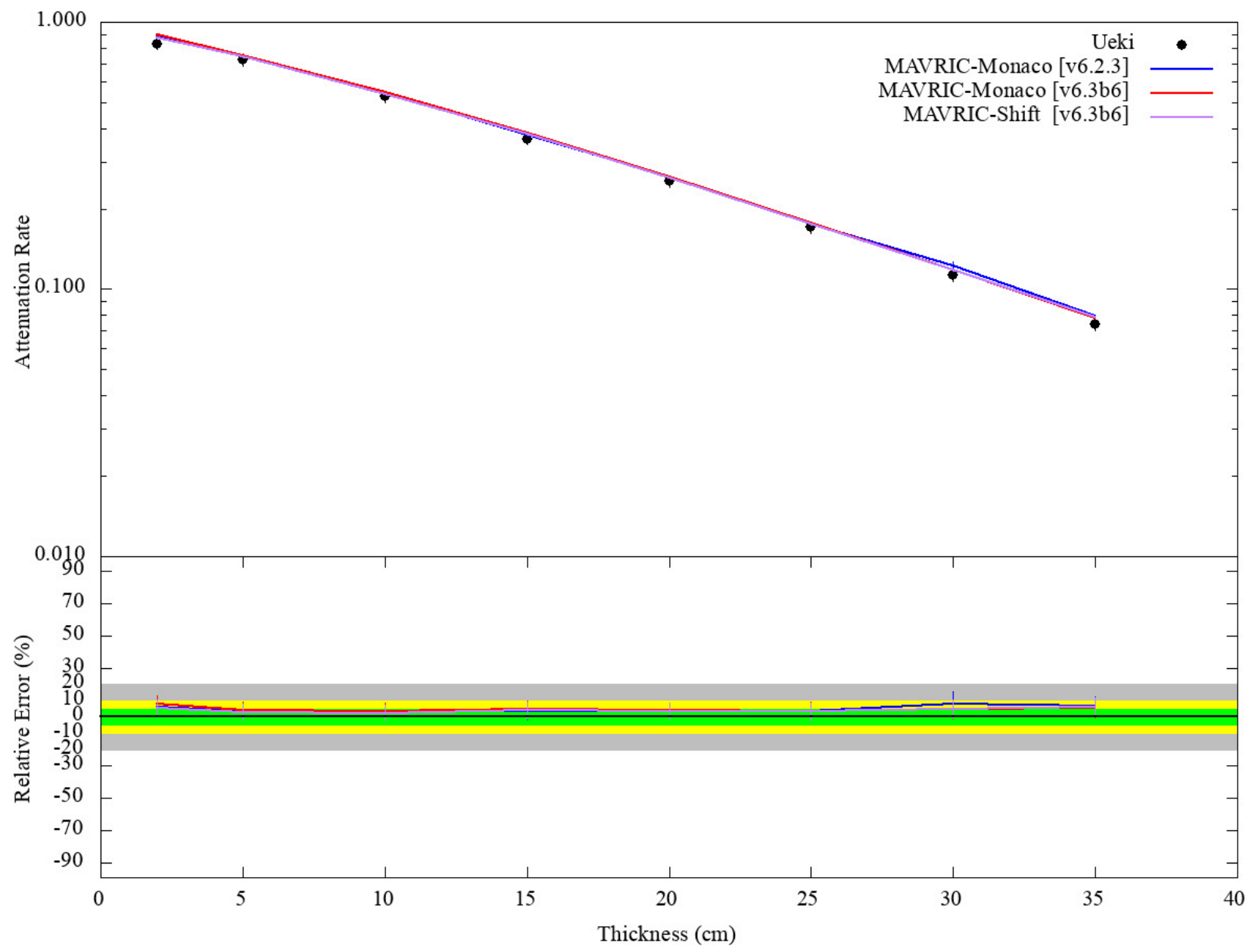

Figure 7. Neutron attenuation rate comparison for the graphite shielding measurement.

Table 4. Timing results for single processor graphite shielding simulations with no shield.

\begin{tabular}{lcccc}
\hline Code & $\begin{array}{c}\text { Number of } \\
\text { processors }\end{array}$ & $\begin{array}{c}\text { CPU time }^{\mathbf{a}} \\
(\mathbf{m i n})\end{array}$ & $\begin{array}{c}\text { Wall time }^{\mathbf{b}} \\
(\mathbf{m i n})\end{array}$ & $\begin{array}{c}\text { FOM }^{\mathbf{c}} \\
(\text { (/min) }\end{array}$ \\
\hline MAVRIC-Monaco [v6.2.3] & 1 & 6 & 6 & $2.98 \mathrm{e} 3$ \\
MAVRIC-Monaco [v6.3b6] & 1 & 4 & 4 & $4.38 \mathrm{e} 3$ \\
MAVRIC-Shift $\quad$ [v6.3b6] & 1 & 5 & 5 & $3.83 \mathrm{e} 3$ \\
\hline
\end{tabular}

${ }^{a}$ Total CPU time by all processors.

b Total execution time.

c Includes both DENOVO and MAVRIC calculations. 


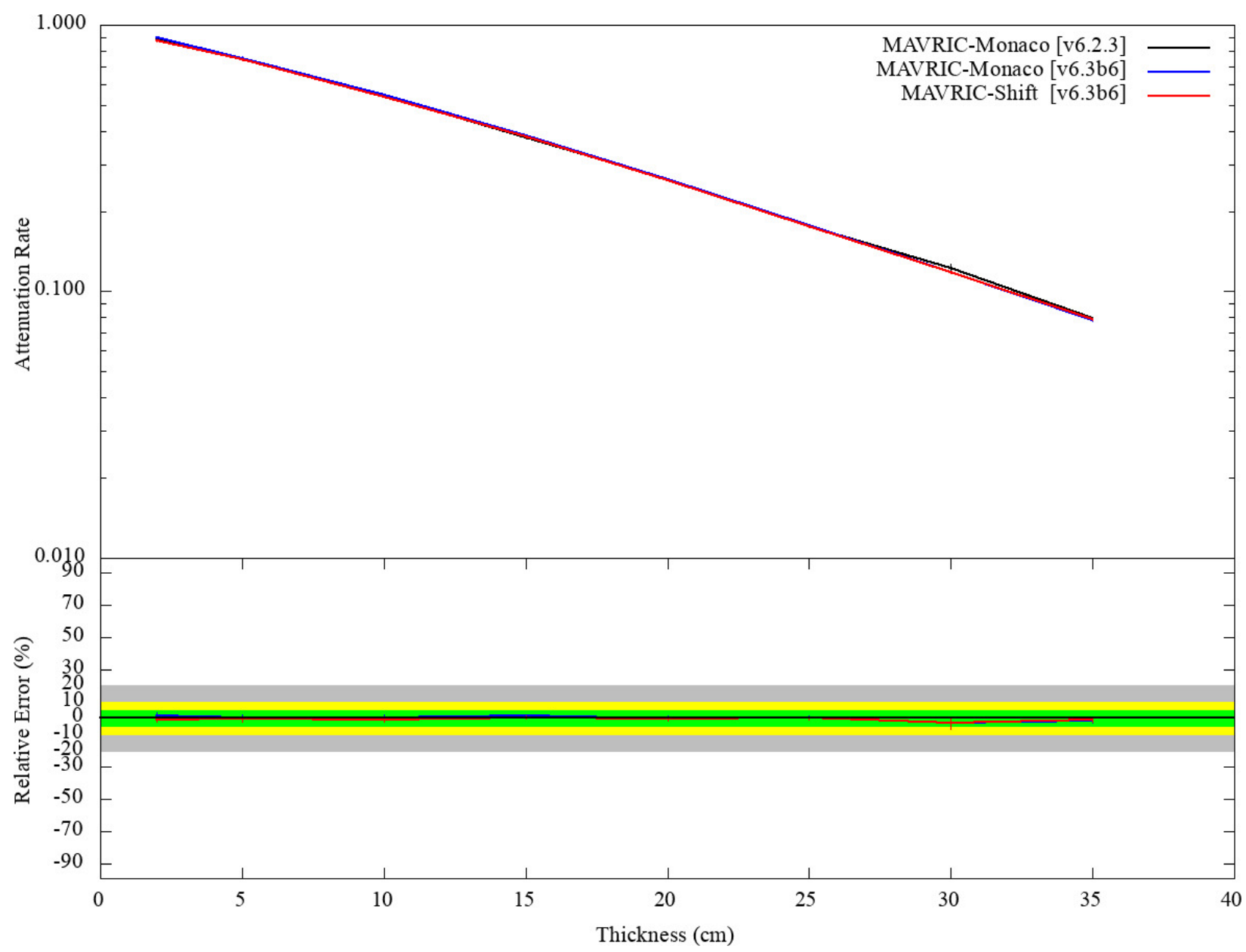

Figure 8. Neutron attenuation rate comparison among codes.

Table 5. Timing results for single processor graphite shielding simulations with $20 \mathrm{~cm}$ graphite shield.

\begin{tabular}{lcccc}
\hline Code & $\begin{array}{c}\text { Number of } \\
\text { processors }\end{array}$ & $\begin{array}{c}\text { CPU time }^{\mathbf{a}} \\
(\mathbf{m i n})\end{array}$ & $\begin{array}{c}\text { Wall time }^{\mathbf{b}} \\
(\mathbf{m i n})\end{array}$ & $\begin{array}{c}\text { FOM }^{\mathbf{c}} \\
(\text { (/min) }\end{array}$ \\
\hline MAVRIC-Monaco [v6.2.3] & 1 & 37 & 37 & $6.57 \mathrm{e} 2$ \\
MAVRIC-Monaco [v6.3b6] & 1 & 38 & 38 & $1.77 \mathrm{e} 3$ \\
MAVRIC-Shift [v6.3b6] & 1 & 52 & 52 & $1.99 \mathrm{e} 3$ \\
\hline
\end{tabular}

${ }^{a}$ Total CPU time by all processors.

b Total execution time.

c Includes both DENOVO and MAVRIC calculations. 
A more comprehensive series of tests was also performed on this model to assess the accuracy and performance of parallel computing provided by MAVRIC-Shift as well as the CADIS approach. An initial number of particles for sampling, $\mathrm{N}=10,000$, was increased quadratically up to 100 times the initial number of particles. Additionally, the simulations were also performed with implicit capture only and with the CADIS method to assess the performance of the variance reduction capability. Furthermore, the number of processors for the MAVRIC-Shift parallel simulations was also increased quadratically up to 16 processors to investigate parallel performance.

Comparison of timing results for serial and parallel simulations are given in Table 6. With parallel computing, the overall runtime improves significantly with increasing numbers of processors. The FOM shows some loss of efficiency due to increased communication between the processors.

Table 6. Timing results for serial and parallel processor graphite shielding simulations with $20 \mathrm{~cm}$ graphite shield and $100 \times N\left(10^{7}\right)$ particles using CADIS.

\begin{tabular}{lccccc}
\hline Code & & $\begin{array}{c}\text { Number of } \\
\text { processors }\end{array}$ & $\begin{array}{c}\text { CPU time }^{\mathbf{a}} \\
\text { (min) }\end{array}$ & $\begin{array}{c}\text { Wall time }^{\mathbf{b}} \\
\text { (min) }\end{array}$ & $\begin{array}{c}\text { FOM }^{\mathbf{c}} \\
\text { (/min) }\end{array}$ \\
\hline \multicolumn{2}{l|}{ MAVRIC-Monaco [v6.3b6] } & 1 & 45 & 45 & $2.73 \mathrm{e} 3$ \\
MAVRIC-Shift & [v6.3b6] & 1 & 88 & 88 & $2.40 \mathrm{e} 3$ \\
MAVRIC-Shift & [v6.3b6] & 4 & 65 & 16 & $3.02 \mathrm{e} 3$ \\
MAVRIC-Shift & [v6.3b6] & 16 & 92 & 6 & $2.21 \mathrm{e} 3$ \\
\hline
\end{tabular}

a Total CPU time by all processors.

b Total execution time.

c Includes both DENOVO and MAVRIC calculations.

A comparison of calculated doses and their relative uncertainties are shown in Fig. 9 through Fig. 12. All results converge to a dose of approximately $0.0130 \mathrm{rem} / \mathrm{h}$. The relative uncertainties improve as the square root of the number of particles, as expected, and are significantly improved with the CADIS approach relative to calculations with implicit capture only. 


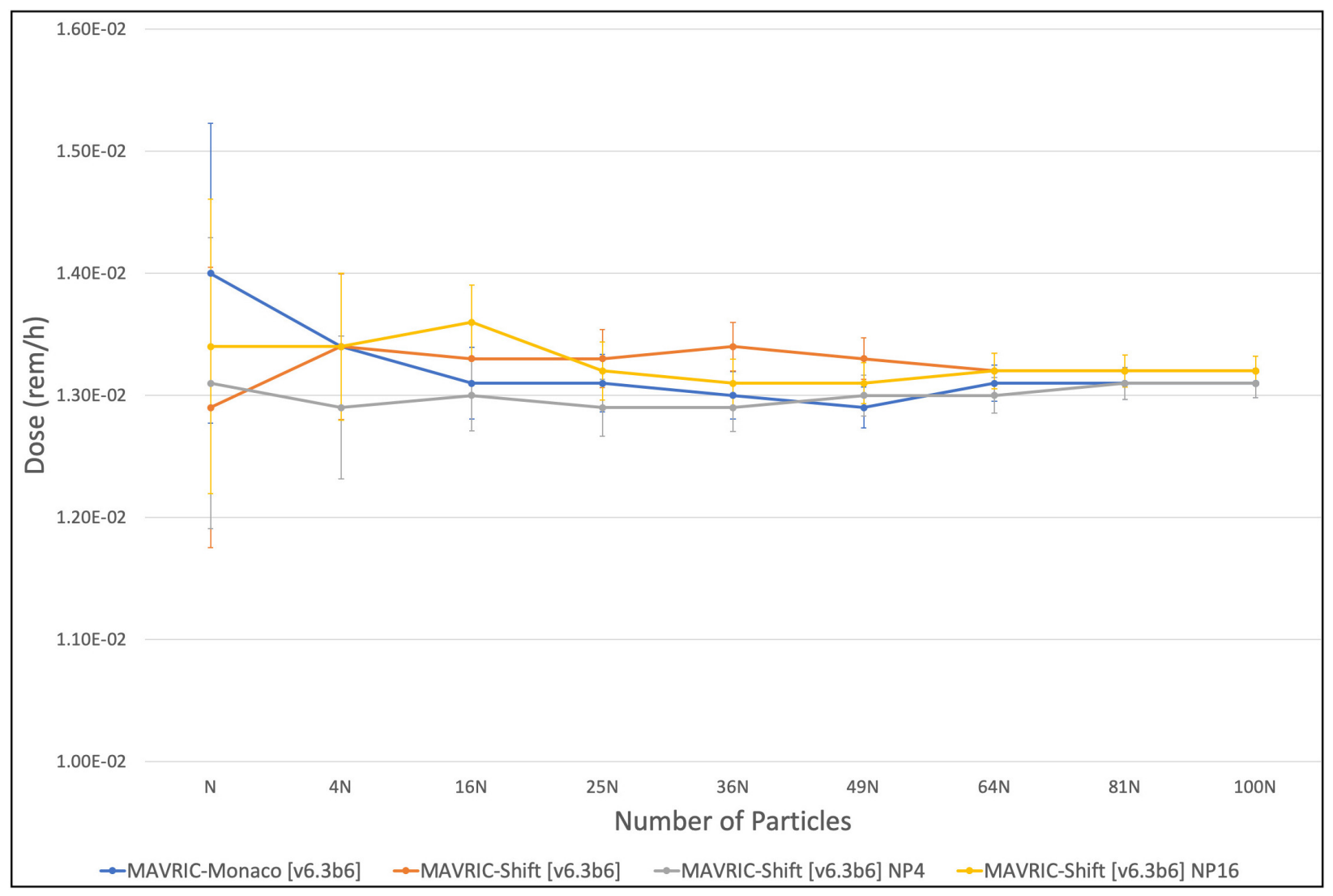

Figure 9. Dose comparison for the graphite shielding measurement via simulations with implicit capture only. 


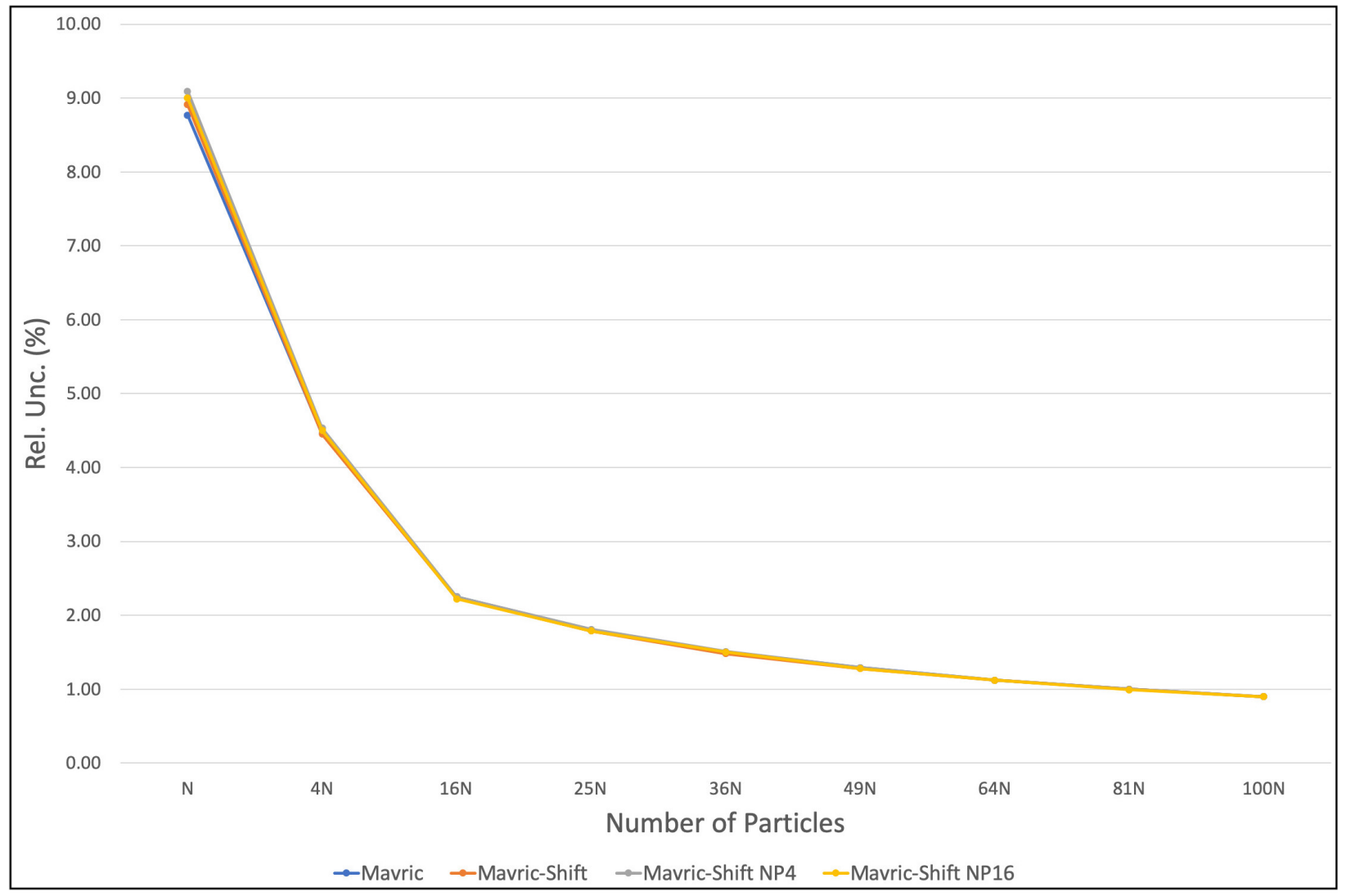

Figure 10. Relative uncertainty comparison for the graphite shielding measurement via simulations with implicit capture only. 


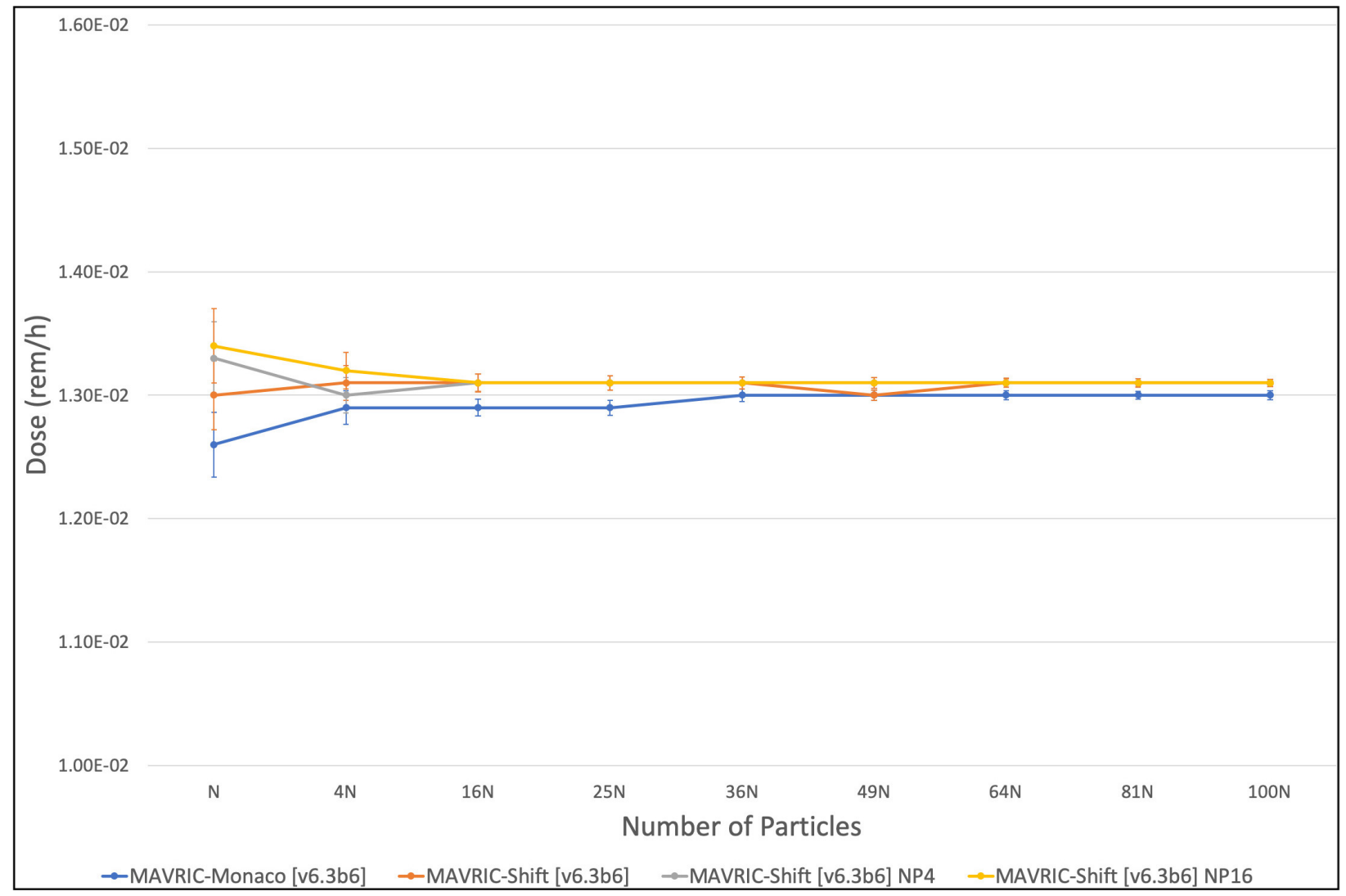

Figure 11. Dose comparison for the graphite shielding measurement via CADIS simulations. 


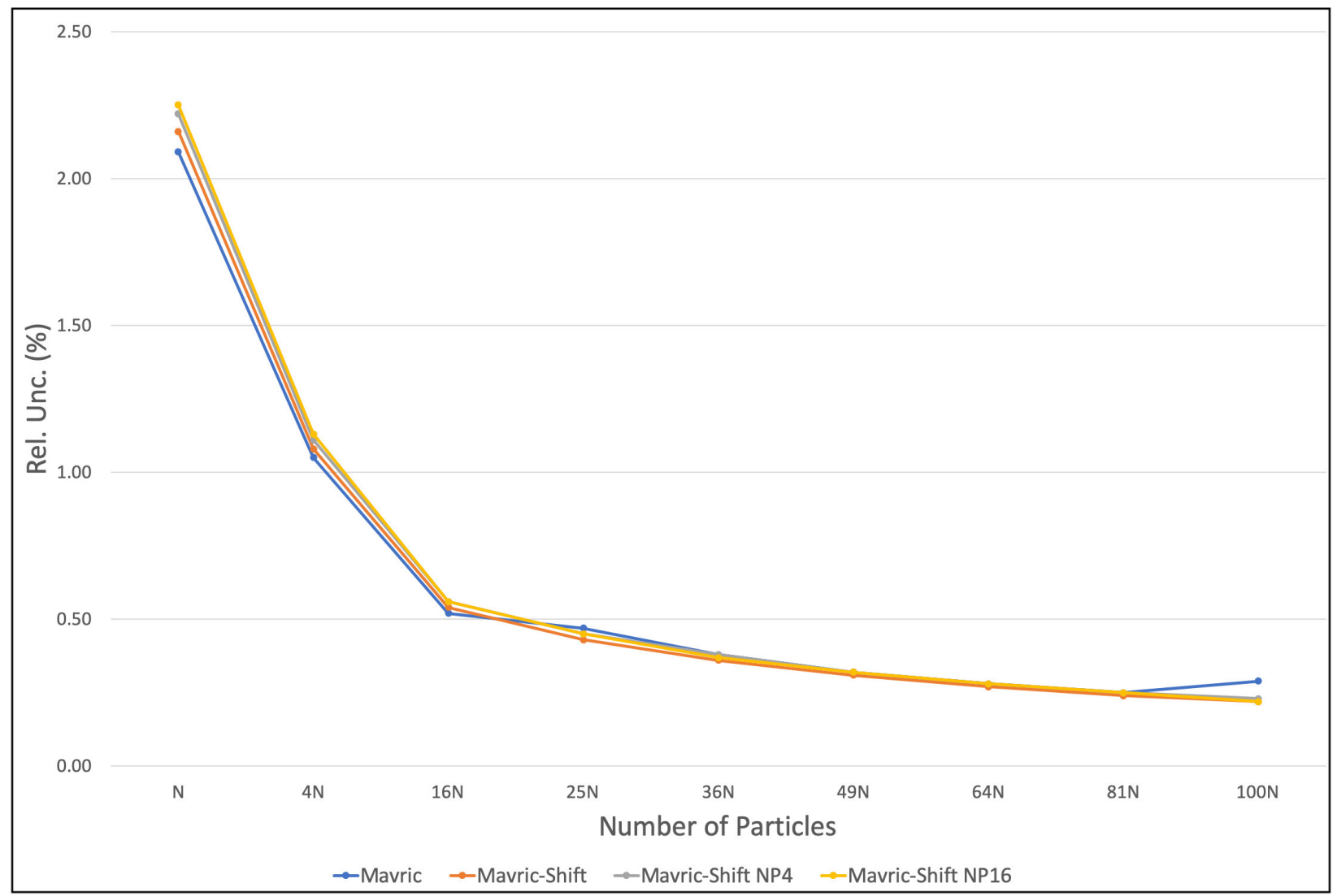

Figure 12. Relative uncertainty comparison for the graphite shielding measurement via CADIS simulations. 
Comparison of the FOM results for serial calculations with only implicit capture, shown in Fig. 13, shows superior performance of MAVRIC-Shift over MAVRIC-Monaco. A similar comparison of the FOM results for serial calculations for the CADIS method, shown in Fig. 14, continues to show a performance degradation due to the more rigorous source methods in Shift relative to Monaco.

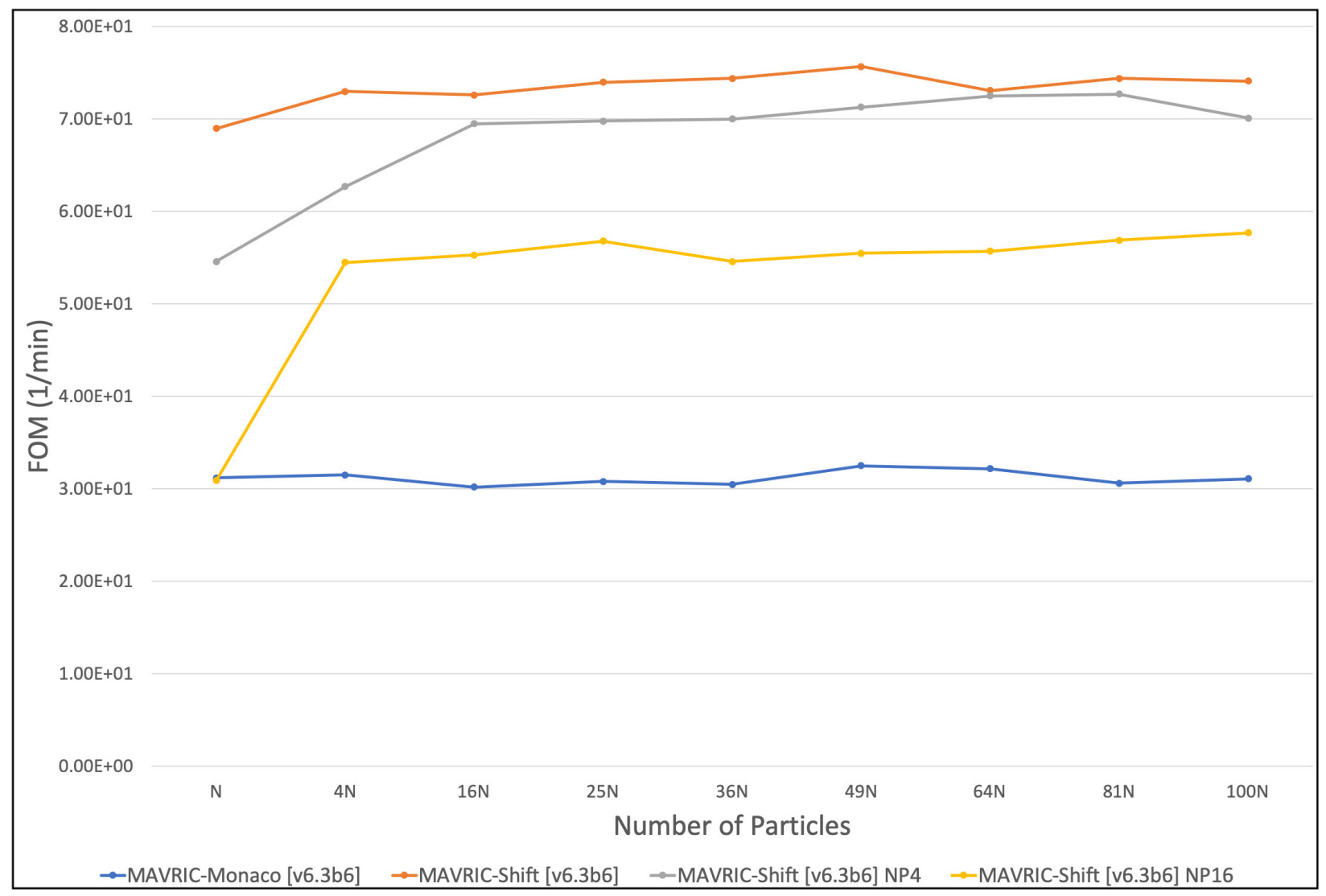

Figure 13. FOM comparison for the graphite shielding measurement via simulations with implicit capture only. 


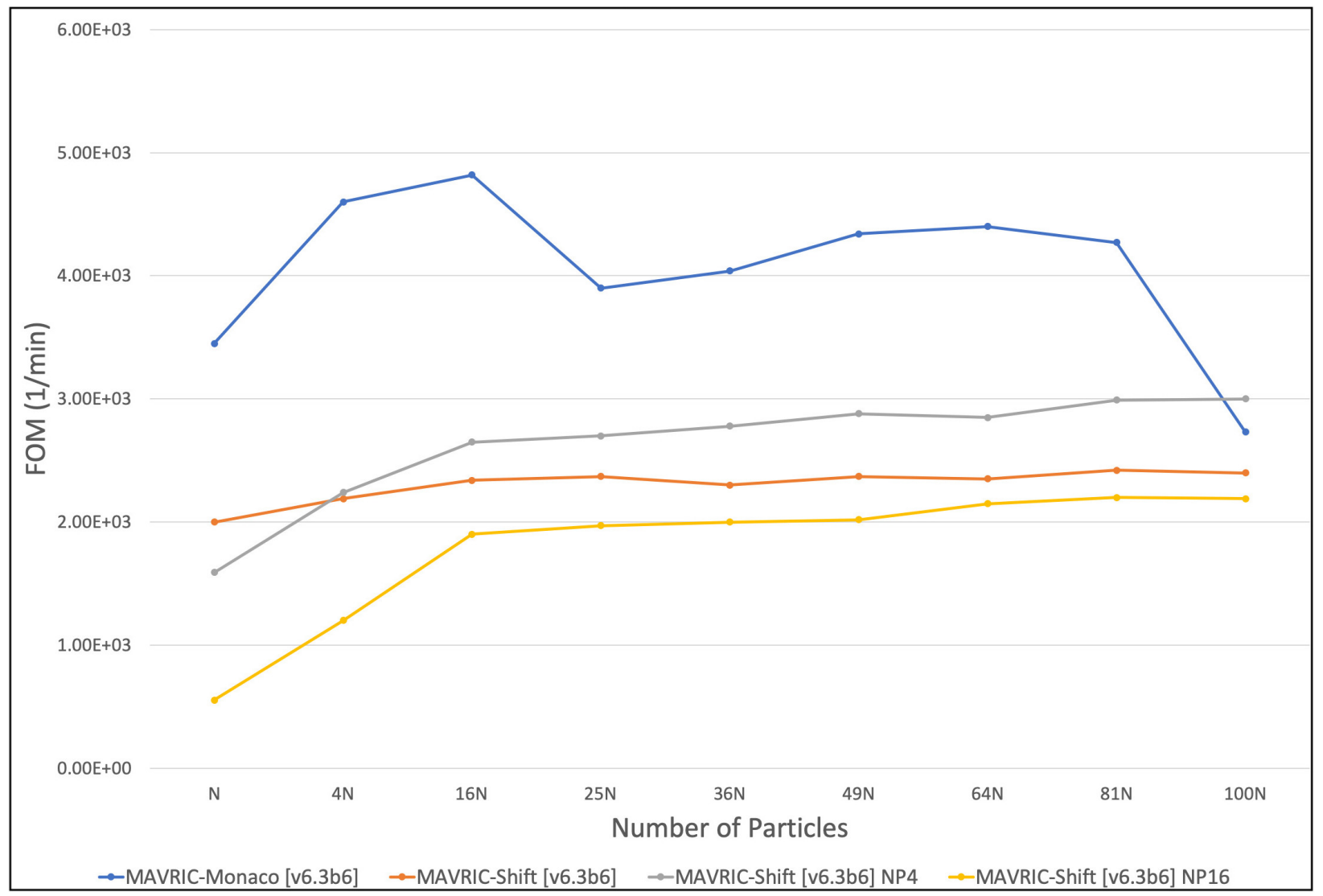

Figure 14. FOM comparison for the graphite shielding measurement via CADIS simulations. 


\subsection{VERIFICATION MODELS}

Another set of models that represent analyses used in practice was used to verify the MAVRIC-Shift method relative to the established MAVRIC-Monaco results. These models mainly focus on SNF transportation and storage applications. As shown in the validation section, MAVRIC-Monaco in the latest stable release (v6.2.3) and the current beta release (v6.3b6) demonstrate good agreement in results. Therefore, only the beta release versions of MAVRIC-Monaco and MAVRIC-Shift codes with ENDF/B-VII.1 nuclear data are used in the verification simulations.

\subsubsection{AOS-100 CASK}

Transportation of radioactive materials is one of the main applications in which estimation of dose rates inside and outside the transported package plays a crucial role in manufacturing, operations, and regulations. A transport packaging system developed by Alpha Omega Services Inc. (AOS) [14], the AOS-100 cask, is considered here to demonstrate and to verify dose rates surrounding the cask. A simplified cask model, shown in Fig. 15, was used in the simulations. The interior of the cask was filled with dry air, and a ${ }^{60} \mathrm{Co}$ gamma source with an activity of $1 \mathrm{Ci}$ was uniformly distributed inside the interior air region.

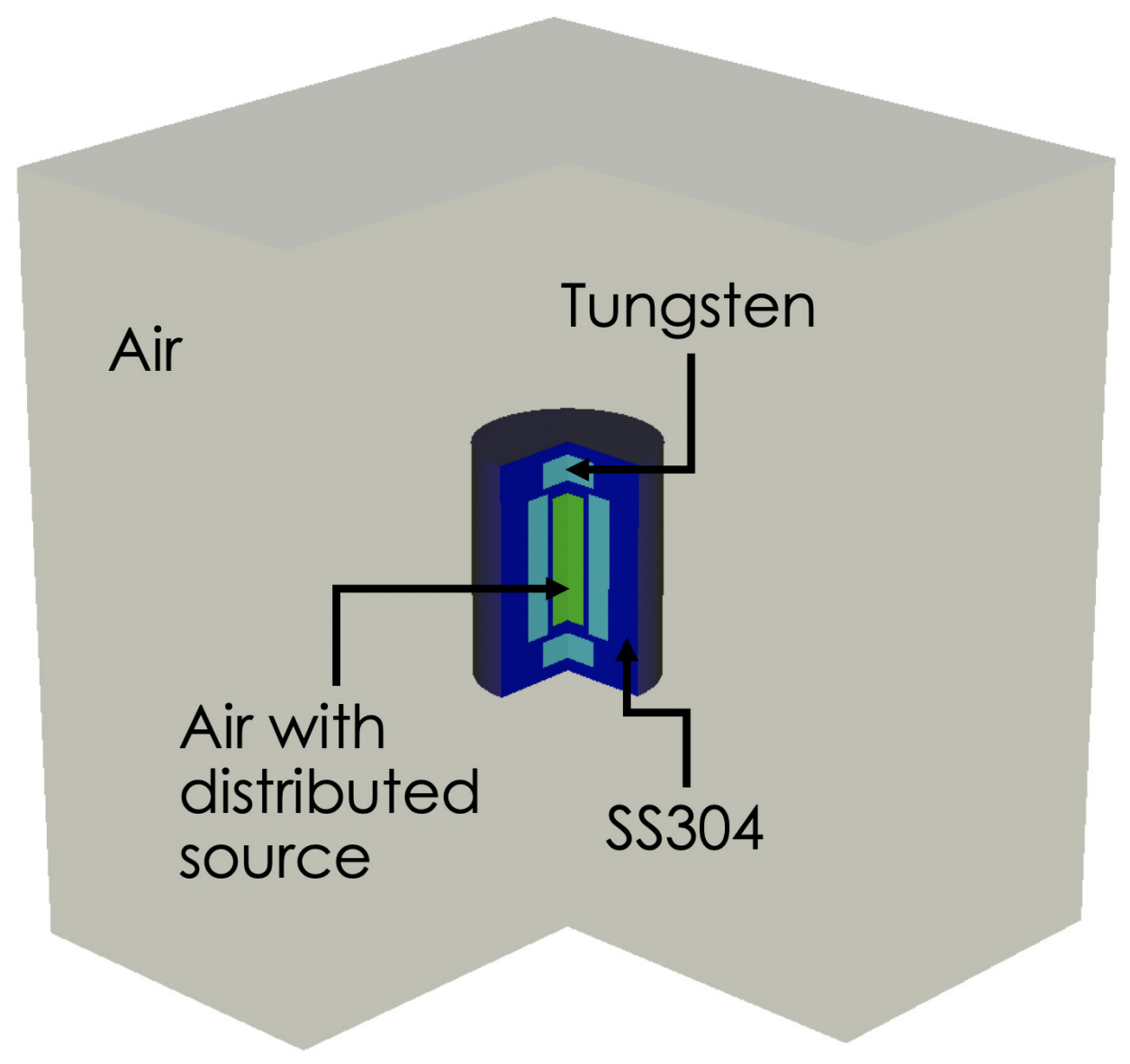

Figure 15. Simplified AOS-100 cask model. 
A mesh tally was used to calculate gamma dose rates outside the cask that was filled with dry air. The FW-CADIS method was used to optimize simulations for the dose rate calculations in which ANSI standard (1977) photon flux-to-dose-rate factors [13] were used as a response in the tally definition. The calculated gamma dose map and the relative uncertainties for the AOS-100 cask model at the central axial plane are shown in Fig. 16. Similarly, the dose rate map and relative uncertainties for the central radial plane are shown in Fig. 17. Both codes produced statistically the same dose rate values within the $10 \%$ relative uncertainties all around the cask. The radial dose rate variation was calculated to be less than $5 \mu \mathrm{rem} / \mathrm{h}$, and the axial dose rates variation was also less than $5 \mu \mathrm{rem} / \mathrm{h}$ in close proximity to the cask's outer surface. Timing results for the codes are listed in Table 7, showing faster simulation time with parallel processing capability in the MAVRIC-Shift simulation with a 16x speedup when using 16 processors.

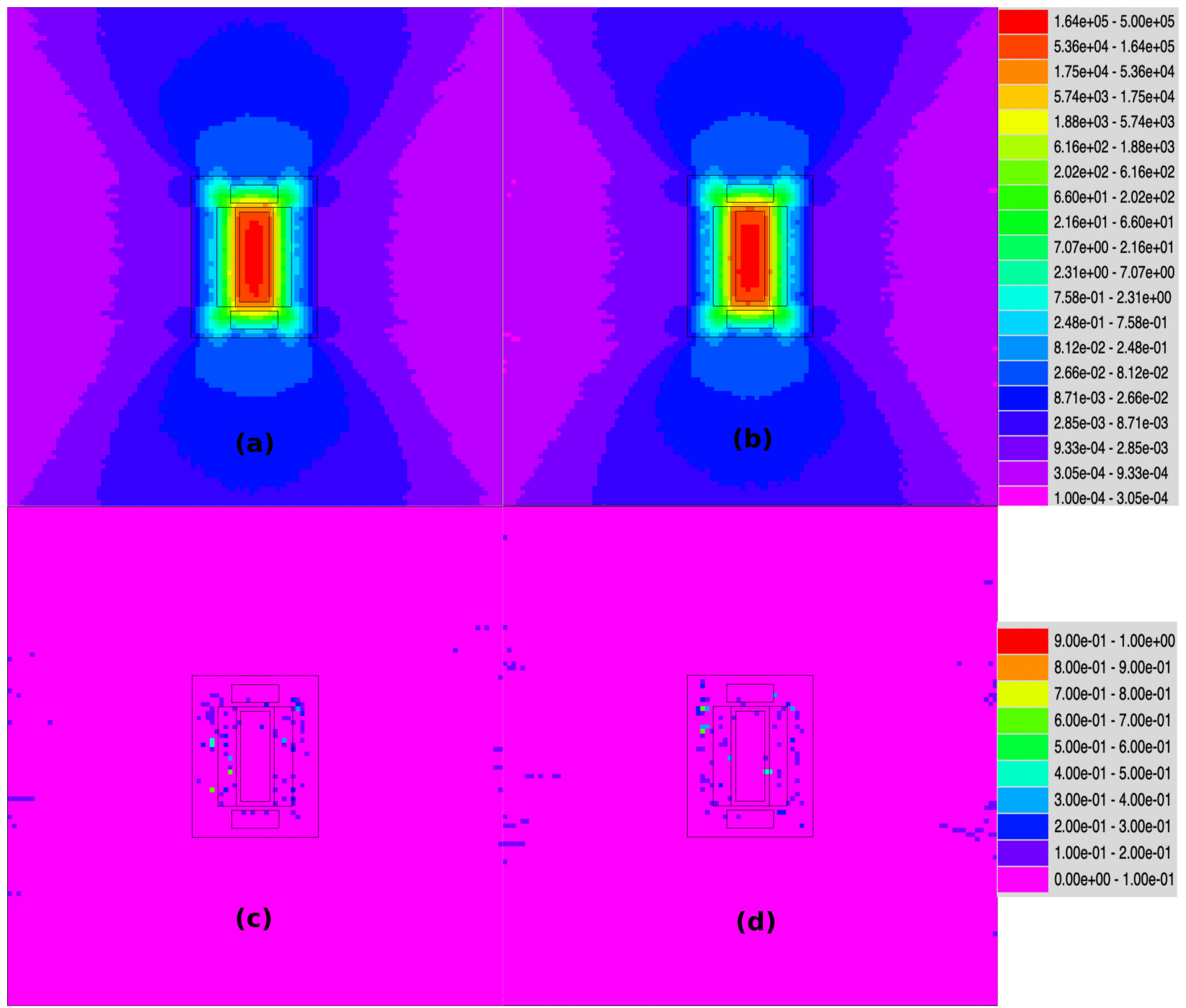

Figure 16. Axial dose rate map (in mrem/h) for the AOS-100 cask model with MAVRIC-Monaco v6.3b6 (a) and MAVRIC-Shift v6.3b6 (b) with their relative uncertainties (c) and (d). 


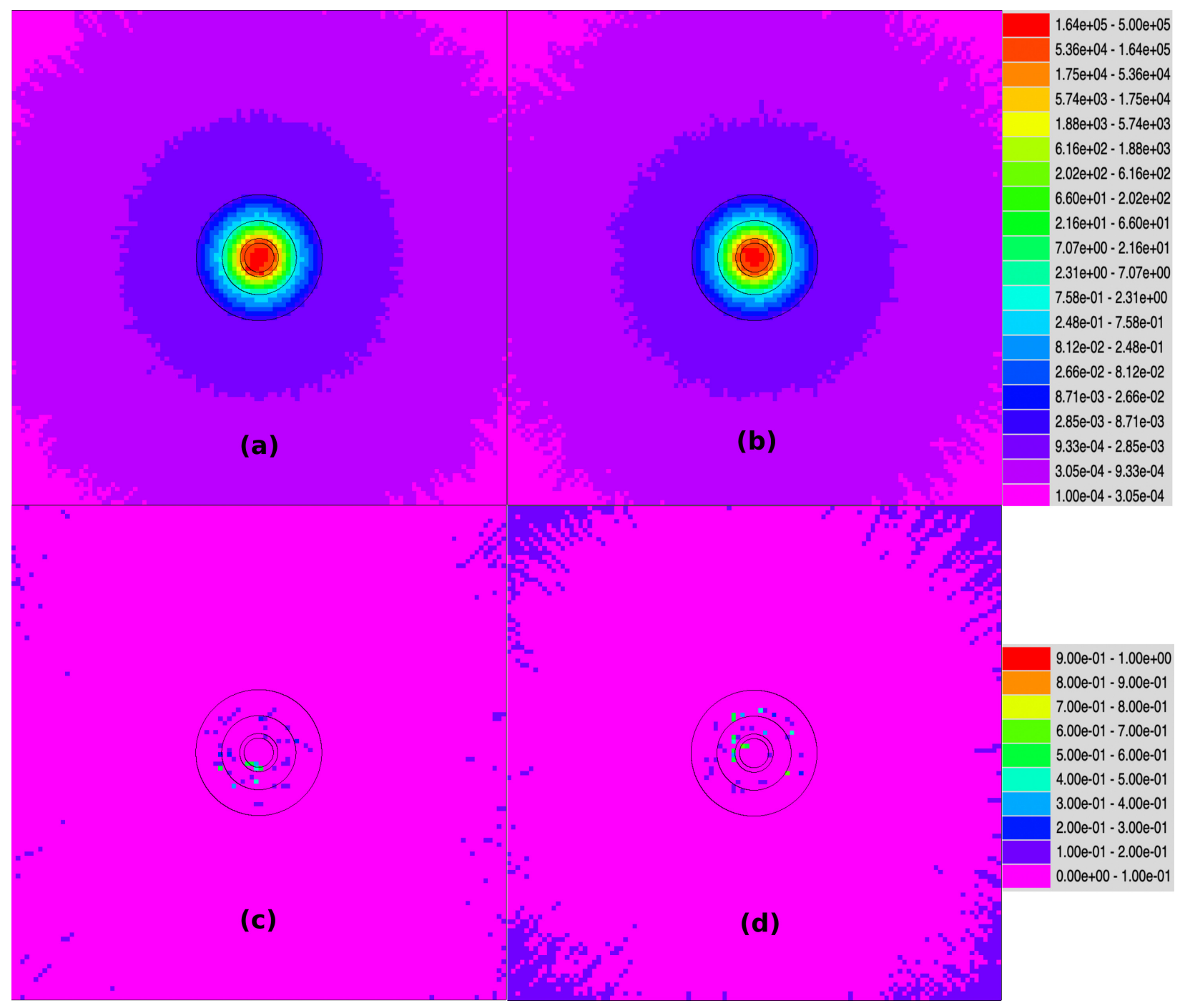

Figure 17. Radial dose rate map (in mrem/h) for the AOS-100 cask model with MAVRIC-Monaco v6.3b6 (a) and MAVRIC-Shift v6.3b6 (b) with their relative uncertainties (c) and (d).

Table 7. Timing results for the AOS-100 cask shielding simulations.

\begin{tabular}{|c|c|c|c|}
\hline Code & $\begin{array}{l}\text { Number of } \\
\text { processors }\end{array}$ & $\begin{array}{l}\text { CPU time } \\
(\text { (min) }\end{array}$ & $\begin{array}{l}\text { Wall time }^{b} \\
(\mathrm{~min})\end{array}$ \\
\hline MAVRIC-Monaco [v6.3b6] ${ }^{\mathrm{d}}$ & 1 & 473 & 473 \\
\hline MAVRIC-Shift $\quad[\mathrm{v} 6.3 \mathrm{~b} 6]^{\mathrm{e}}$ & 16 & 470 & 29 \\
\hline
\end{tabular}

a Total CPU time by all processors.

b Total execution time. 


\subsubsection{TN-24P CASK}

TN-24P is a SNF cask for transportation and storage developed by Transnuclear, Inc. This model was previously studied by Wiarda et al. [15]. TN-24P contains spent fuel assemblies and has a steel body for shielding gammas and a hydrogenous resin layer to shield neutrons. A simplified version of the TN-24P cask model was used in the simulations as shown in Fig. 18. Spent fuel assemblies were homogenized for simplicity - shown as $\mathrm{W}$ and $\mathrm{V}$ assemblies in the figure - and were filled with fresh uranium dioxide fuel (3\% enriched) for verification purposes. Neutron and gamma sources were uniformly distributed in each $\mathrm{W}$ and $\mathrm{V}$ assembly in the model. In addition, a ${ }^{60} \mathrm{Co}$ gamma source was also uniformly distributed in both top and bottom nozzles, as well as in the plenum of the cask to simulate hardware activation. All the surrounding region of the cask was filled with dry air.

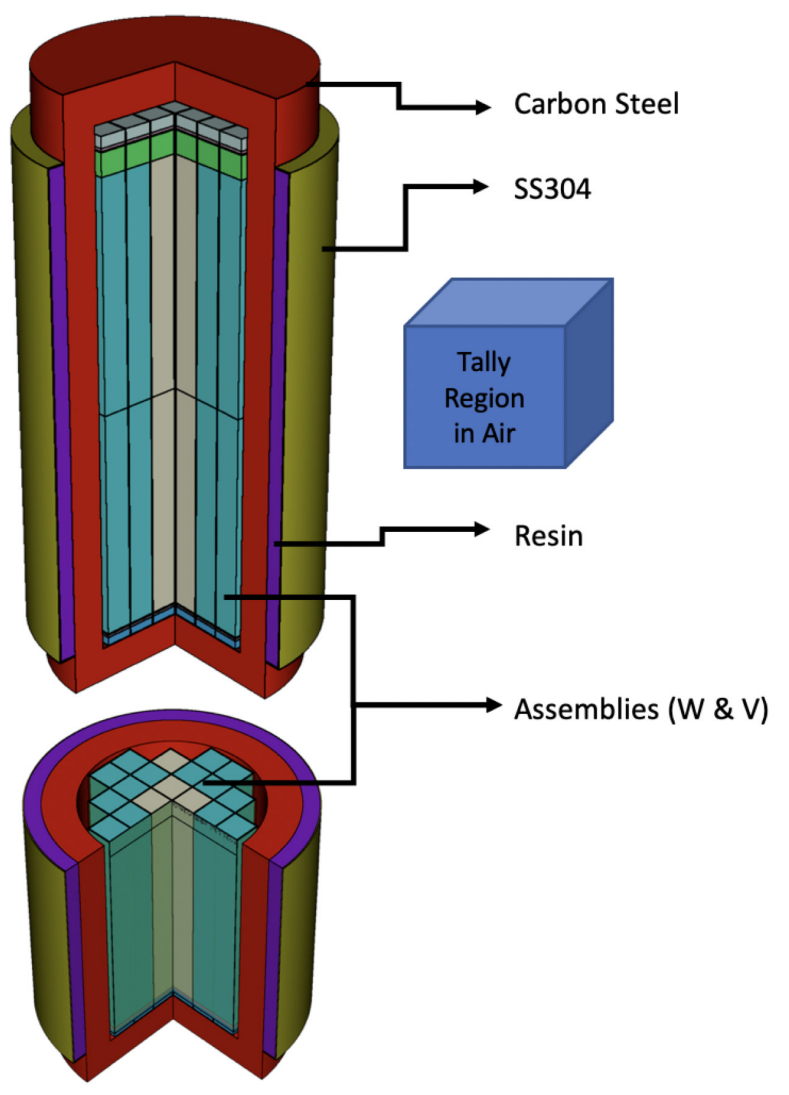

Figure 18. Simplified TN-24P cask model.

A region tally was used to calculate gamma dose over $1 \mathrm{~m}^{3}$ region by using the ANSI standard (1977) photon flux-to-dose-rate factors. The region tally was around $1.5 \mathrm{~m}$ away from the cask's surface and was centered at the mid-height of the cask. The CADIS variance reduction method was employed to optimize the dose values in the region tally. Calculated dose values in the region tally from both MAVRIC-Monaco and MAVRIC-Shift codes are in good agreement and are given in Table 8. Both codes perform similarly with an equal amount of computational resources, but the MAVRIC-Shift code was able to finish simulations in a fraction of the time required for simulations with the MAVRIC-Monaco with its parallel computing capability. The FOM was calculated in the region tally for the dose rate results. Timing comparison of the simulations is given in Table 9. 
Table 8. Calculated dose values for the TN-24P cask shielding simulations.

\begin{tabular}{|cc|cc|c|}
\hline \multicolumn{2}{|c|}{ MAVRIC-Monaco [v6.3b6] } & \multicolumn{2}{|c|}{ MAVRIC-Shift [v6.3b6] } & Delta \\
\hline $\begin{array}{c}\text { dose } \\
(\mathbf{m r e m} / \mathbf{h})\end{array}$ & $\begin{array}{c}\text { relative } \\
\text { uncert. }(\%)\end{array}$ & $\begin{array}{c}\text { dose } \\
(\mathbf{m r e m} / \mathbf{h})\end{array}$ & $\begin{array}{c}\text { relative } \\
\text { uncert. }(\%)\end{array}$ & $\begin{array}{c}\text { Shift/Monaco - 1 } \\
(\%)\end{array}$ \\
\hline 6.49 & 0.41 & 6.47 & 0.76 & -0.38 \\
\hline
\end{tabular}

Table 9. Timing results for the $\mathrm{TN}-24 \mathrm{P}$ cask shielding simulations.

\begin{tabular}{|c|c|c|c|c|}
\hline Code & $\begin{array}{l}\text { Number of } \\
\text { processors }\end{array}$ & $\begin{array}{l}\text { CPU time } \\
\quad(\min )\end{array}$ & $\begin{array}{l}\text { Wall time } \\
(\mathrm{min})\end{array}$ & $\begin{array}{l}\text { FOMc } \\
\text { (/min) }\end{array}$ \\
\hline MAI & 1 & 283 & 283 & $2.12 \mathrm{e} 2$ \\
\hline MAVRIC-Shift & 16 & 384 & 24 & $4.52 \mathrm{e} 1$ \\
\hline
\end{tabular}

a Total CPU time by all processors.

b Total execution time. 


\subsubsection{MPC-32 CANISTER}

A model representative of an SNF transportation package developed by Holtec International [16] was also modeled. A simplified version of a HI-STAR 100 (MPC-32) canister with a fictitious radioactive source representative of non-decayed irradiated fuel was placed in geometry representative of Westinghouse $17 \times 17$ pressurized water reactor (PWR) fuel assemblies, which were placed in the canister. The MPC-32 model used in the simulations is shown in Fig. 19. More than 1,000 neutron and gamma source distributions were modeled in the fuel assemblies, providing a representative radial and axial source distributions in the canister.

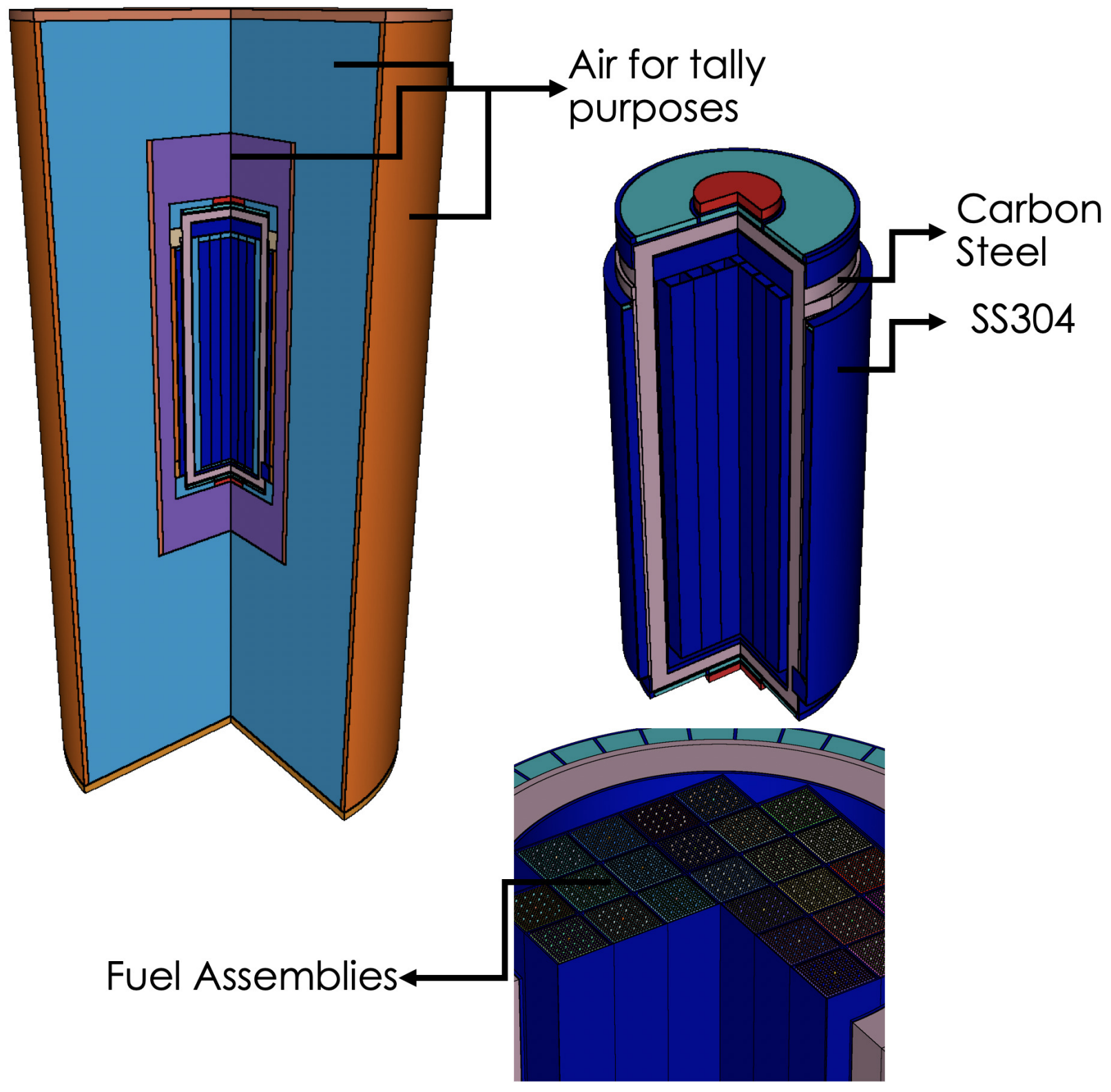

Figure 19. Simplified MPC-32 canister model. 
Two mesh tallies, one for neutrons and one for gammas, were used to calculate the neutron and gamma dose rates outside the canister, which was surrounded by dry air. A MAVRIC utility (mtBinOp) was also used to post-process neutron and gamma dose rates and to calculate the total dose rate by summing both. The FW-CADIS method was used to optimize simulations for the dose rate calculations in which ANSI standard (1977) neutron and photon flux-to-dose-rate factors were used as responses in the corresponding tally definitions.

Calculated axial neutron dose rate maps at the center plane of the canister and their relative uncertainties are shown in Fig. 20 for MAVRIC-Monaco and MAVRIC-Shift. Calculated radial neutron dose rate maps at the center plane of the canister and their relative uncertainties are also shown in Fig. 21. Similarly, calculated gamma dose rate maps for the model are shown in Fig. 22 and Fig. 23. Finally, total dose rate maps (sum of neutron and gamma doses) are shown in Fig. 24. In all cases, MAVRIC-Shift simulations yielded very similar dose rate values compared to those of MAVRIC-Monaco simulations, with slightly lower uncertainties for identical model parameters, proving to be a slightly more effective simulation tool for this model. Total dose rates around the cask were dominated by the gamma dose, reaching up to 300 $\mathrm{mrem} / \mathrm{h}$ at $2 \mathrm{~m}$ away from the canister's surface. Multiple processors have been used with the MAVRICShift simulations and overall simulation time is shorter than that of MAVRIC-Monaco as can be seen in Table 10. MAVRIC-Shift performs more rigorous source discretization and biasing for all the available sources and responses in the problem, ensuring a more accurate source representation in the FW-CADIS method. As a result, the time required for simulations increases linearly with the number of sources and responses in the model. Optimization of the source discretization and biasing will be investigated further as Shift development continues.

Table 10. Timing results for the MPC-32 canister shielding simulations.

\begin{tabular}{|c|c|c|c|}
\hline Code & $\begin{array}{l}\text { Number of } \\
\text { processors }\end{array}$ & $\begin{array}{c}\text { CPU time } \\
(\text { min })\end{array}$ & $\begin{array}{l}\text { Wall time } \\
(\mathrm{min})\end{array}$ \\
\hline MAVRIC-Monaco [v6.3b6] & 1 & 312 & 312 \\
\hline MAVRIC-Shift [v6.3b6] & 256 & 12,831 & 50 \\
\hline
\end{tabular}




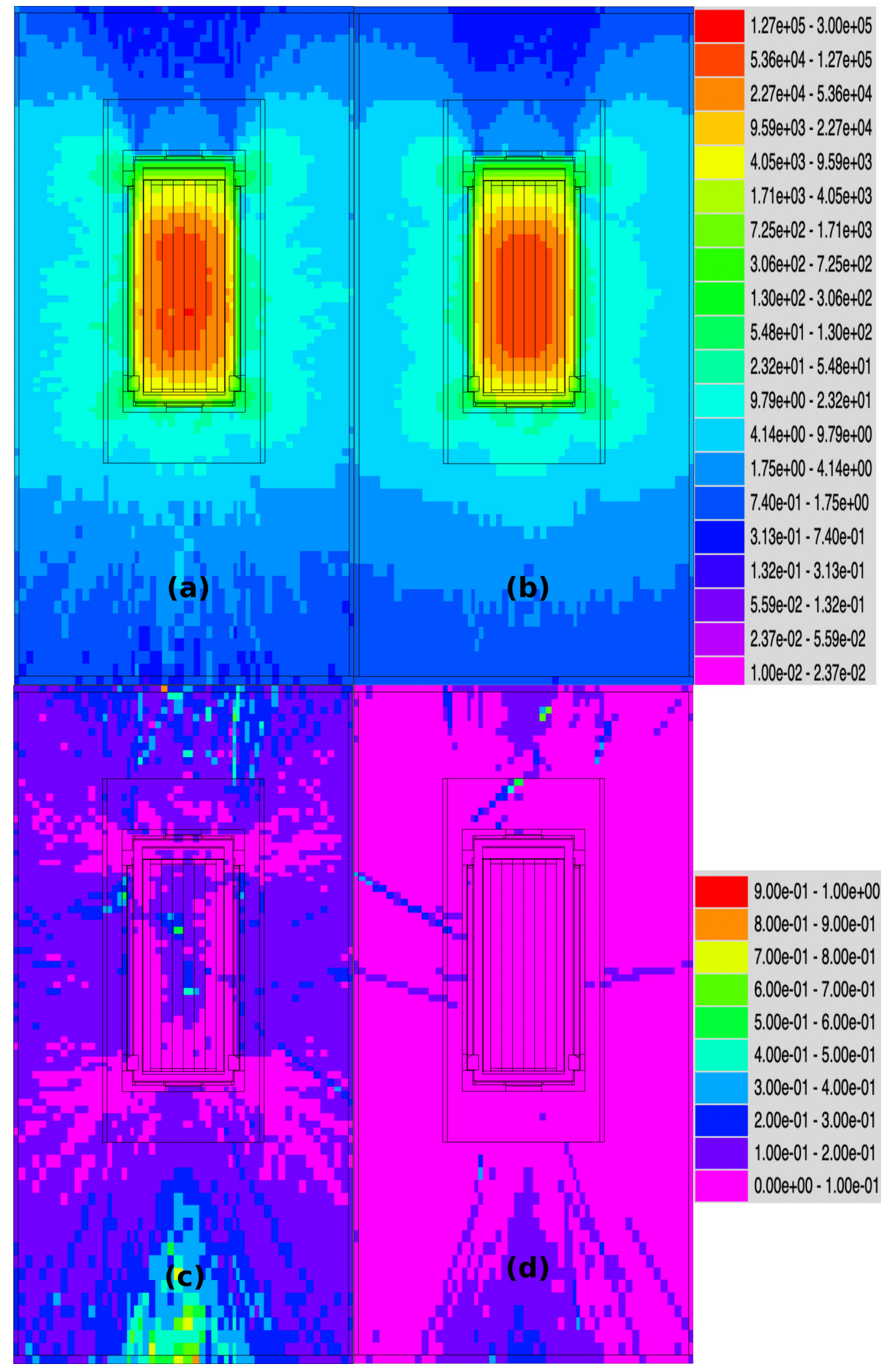

Figure 20. Axial neutron dose rate map (in mrem/h) for the MPC-32 cask model with MAVRICMonaco v6.3b6 (a) and MAVRIC-Shift v6.3b6 (b) with their relative uncertainties (c) and (d). 


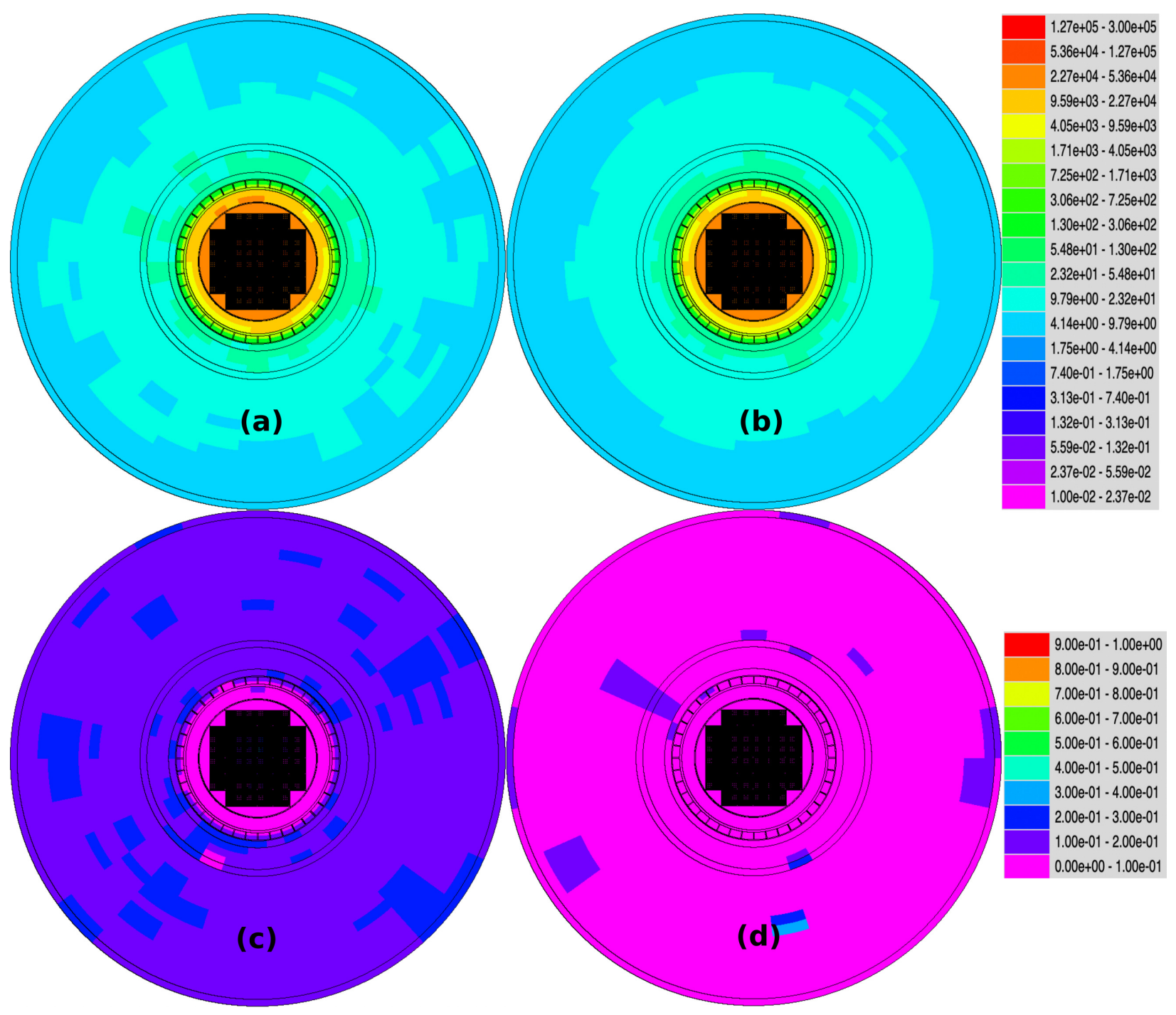

Figure 21. Radial neutron dose rate map (in mrem/h) for the MPC-32 cask model with MAVRICMonaco v6.3b6 (a) and MAVRIC-Shift v6.3b6 (b) with their relative uncertainties (c) and (d). 


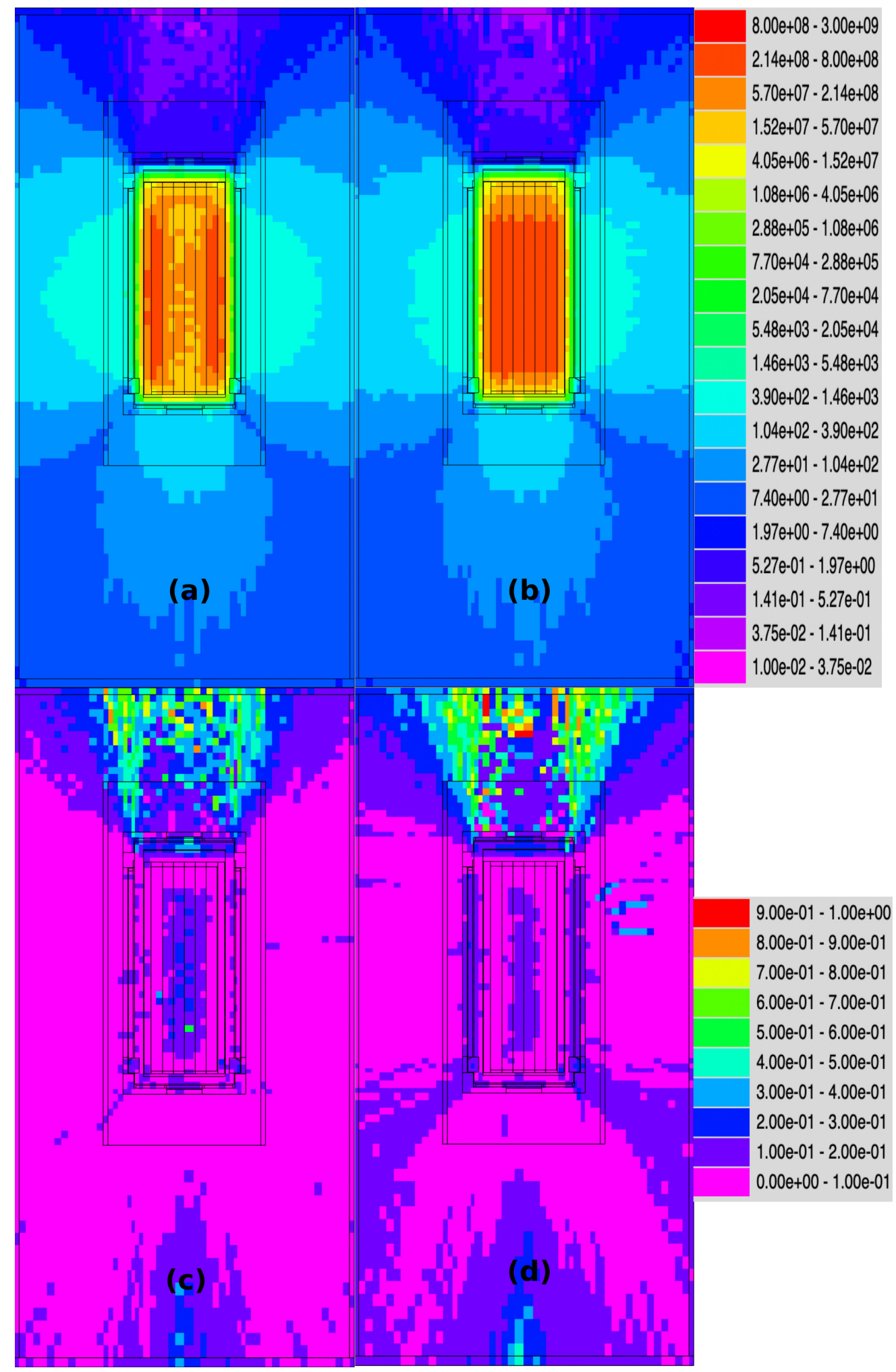

Figure 22. Axial gamma dose rate map (in $\mathrm{mrem} / \mathrm{h}$ ) for the MPC-32 cask model with MAVRICMonaco v6.3b6 (a) and MAVRIC-Shift v6.3b6 (b) with their relative uncertainties (c) and (d). 


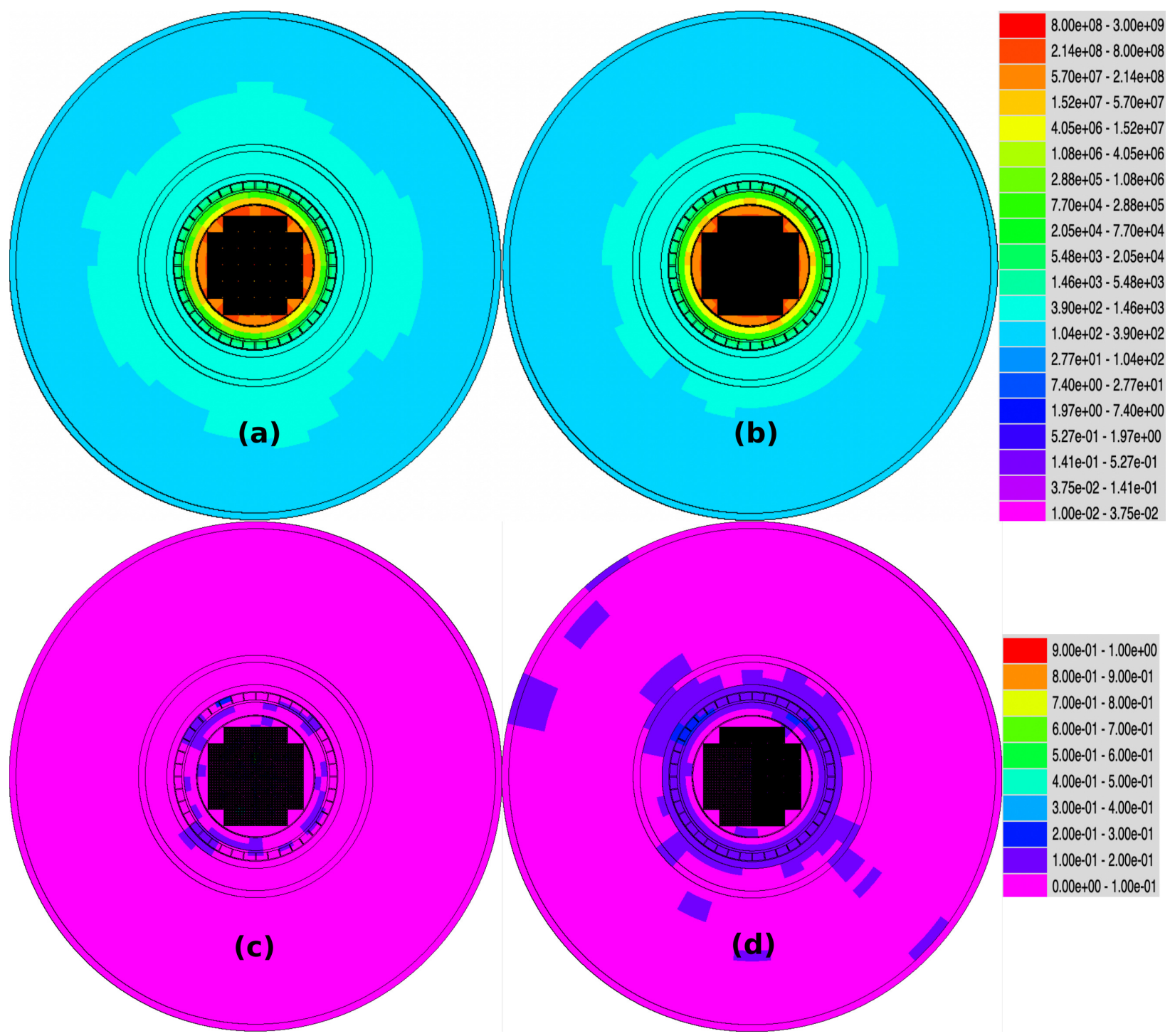

Figure 23. Radial gamma dose rate map (in mrem/h) for the MPC-32 cask model with MAVRICMonaco v6.3b6 (a) and MAVRIC-Shift v6.3b6 (b) with their relative uncertainties (c) and (d). 


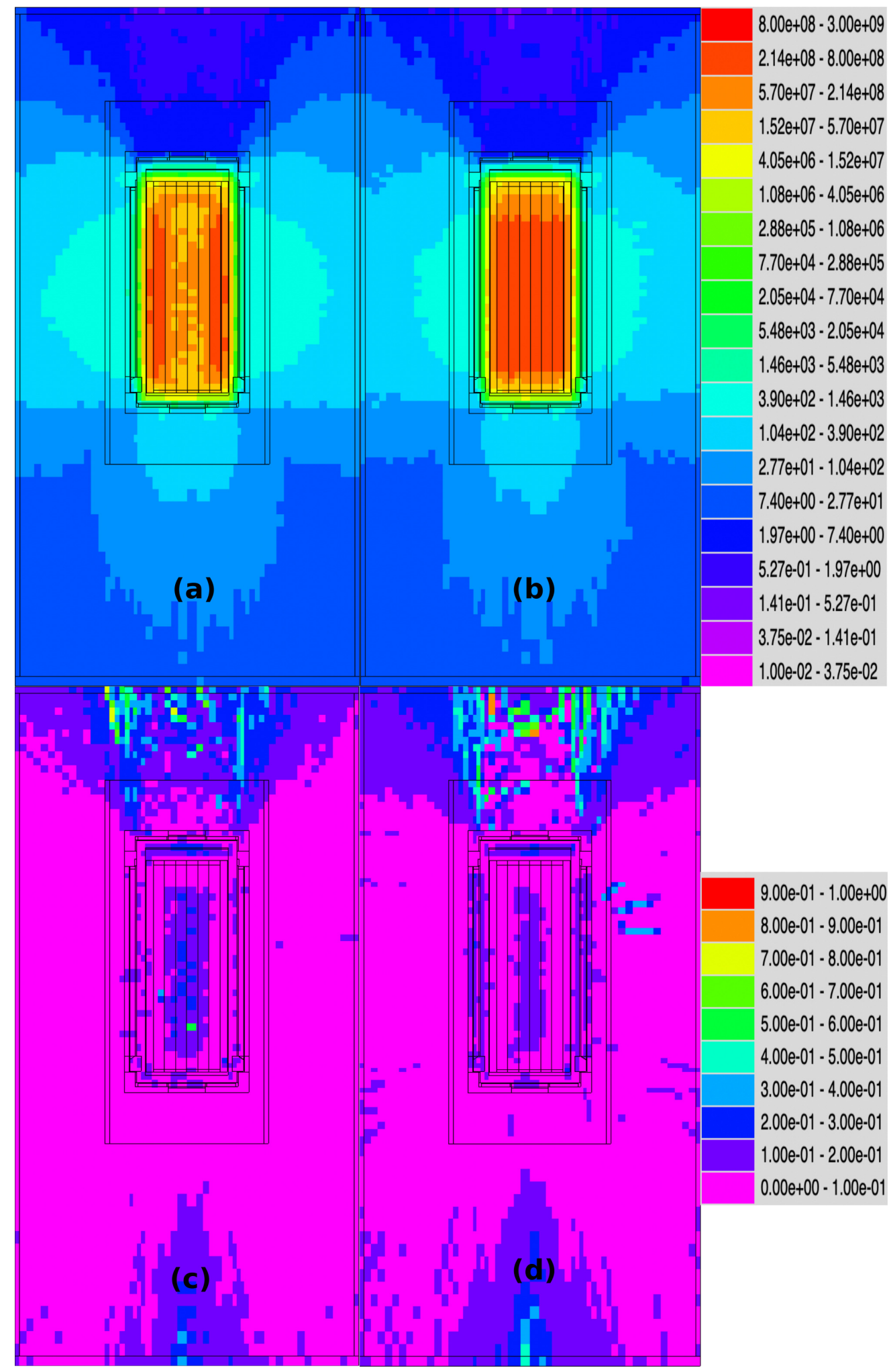

Figure 24. Axial total dose rate map (in $\mathrm{mrem} / \mathrm{h}$ ) for the MPC-32 cask model with MAVRIC-Monaco v6.3b6 (a) and MAVRIC-Shift v6.3b6 (b) with their relative uncertainties (c) and (d). 


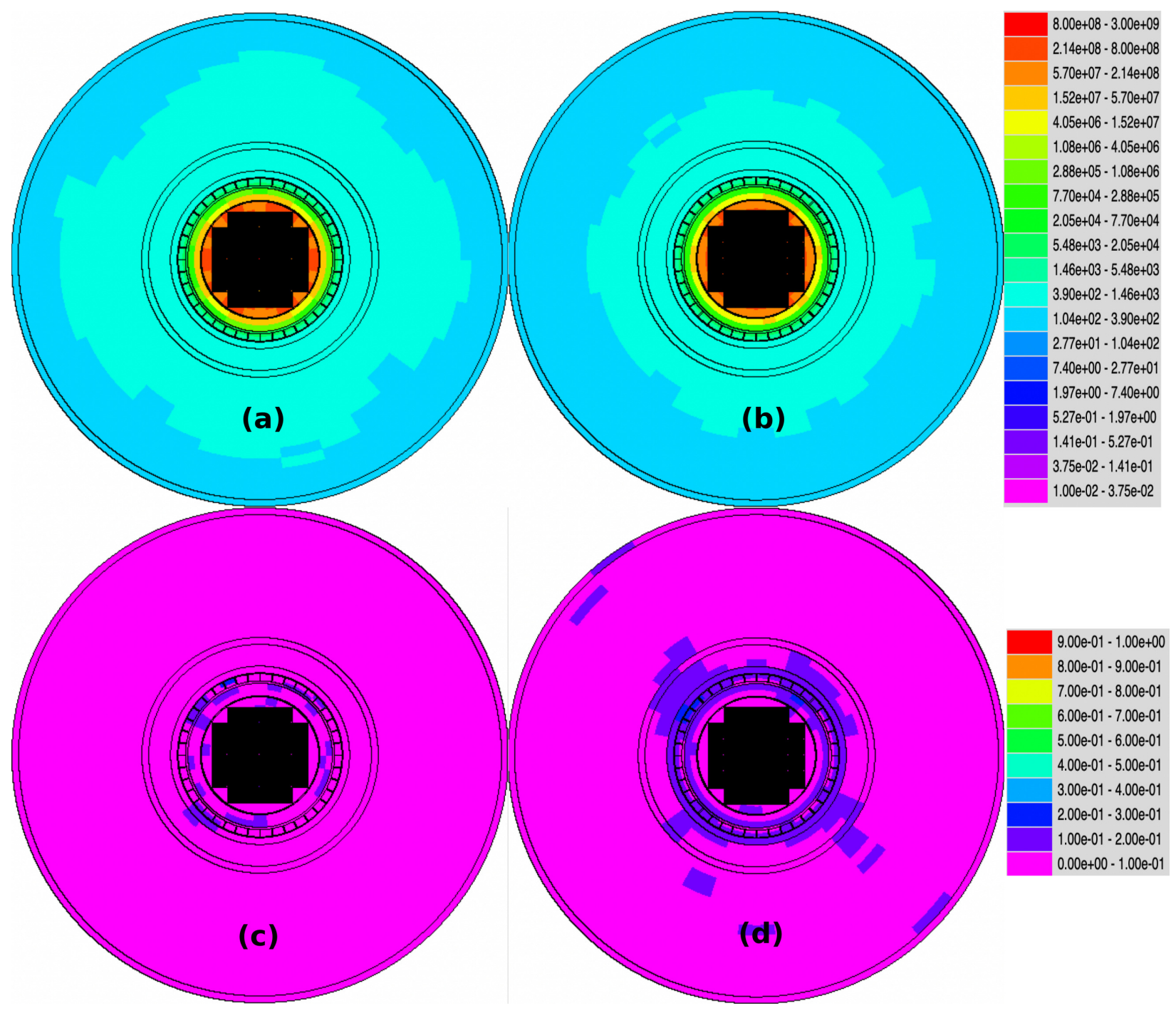

Figure 25. Radial total dose rate map (in $\mathrm{mrem} / \mathrm{h}$ ) for the MPC-32 cask model with MAVRIC-Monaco v6.3b6 (a) and MAVRIC-Shift v6.3b6 (b) with their relative uncertainties (c) and (d). 


\subsubsection{CANISTER WITH DETAILED SOURCE DEFINITIONS}

A more realistic canister, which is similar to the MPC-32 canister, was modeled for verification purposes with very detailed source distributions in the fuel elements, representative of a model generated with UNFST\&DARDS models, as shown in Fig. 26. The canister model contains boiling water reactor (BWR) SNF assemblies which have pin-by-pin geometry representation and detailed neutron and photon axial source distributions. There are more than 1,000 neutron sources and around 900 gamma sources distributed in the model. The combined neutron and gamma dose rate was calculated with the FW-CADIS method to optimize a cylindrical mesh tally which was using the ANSI standard (1977) neutron and photon flux-todose-rate factors as responses.
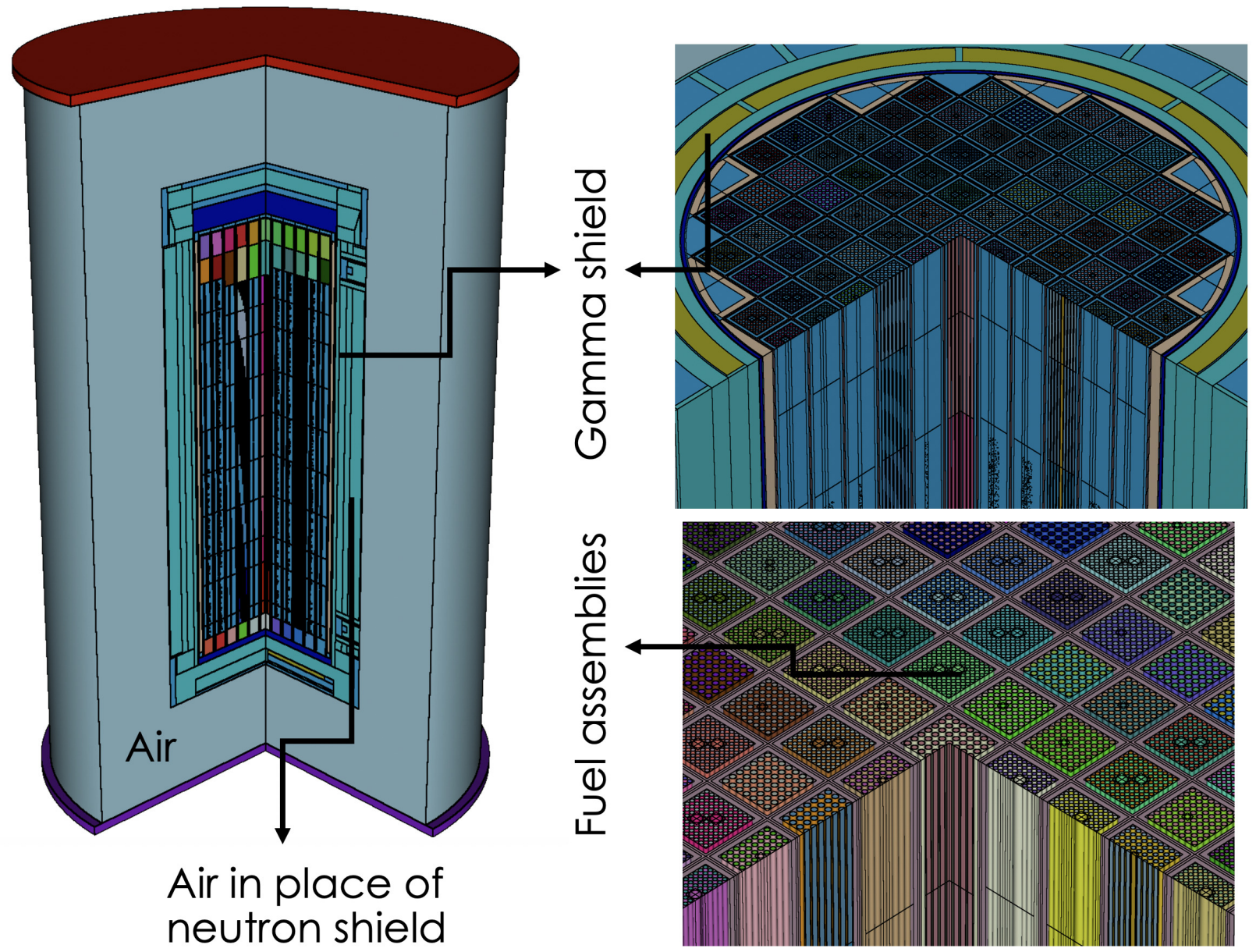

Figure 26. Simplified canister model with detailed source definitions.

A comparison of the axial distribution of the total dose rate around $1 \mathrm{~m}$ away from the canister is shown in Fig. 27. Calculated dose rates from MAVRIC-Monaco and MAVRIC-Shift agree well within 10\% relative uncertainties. Timing comparison of the simulations is also given in Table 11 and shows similar trend in performance as the previous MAVRIC-Shift simulation of the MPC-32 canister model. 


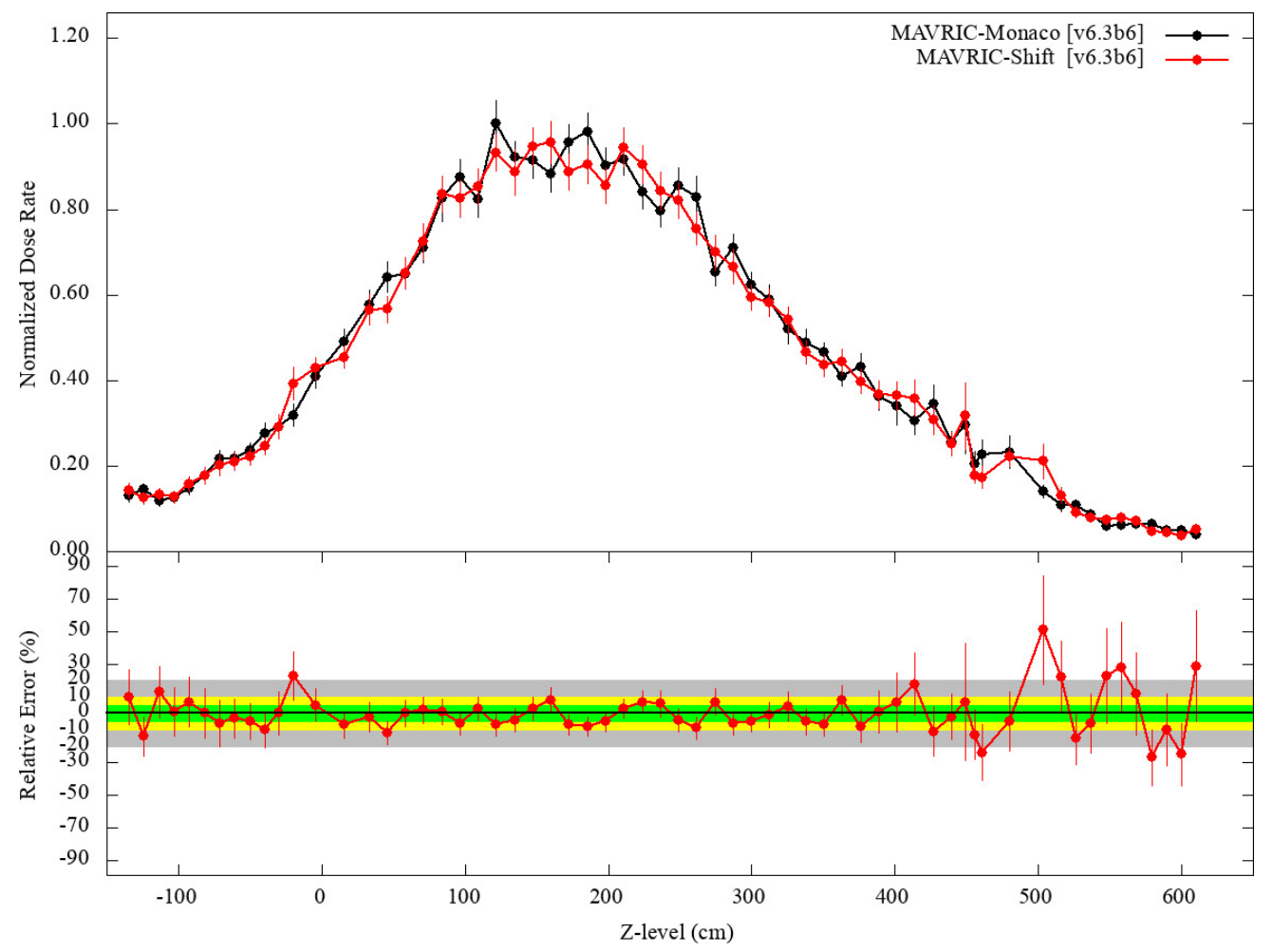

Figure 27. Neutron flux comparison between codes.

Table 11. Timing results for the canister shielding simulations.

\begin{tabular}{|c|c|c|c|}
\hline Code & $\begin{array}{l}\text { Number of } \\
\text { processors }\end{array}$ & $\begin{array}{c}\text { CPU time } \\
(\min )\end{array}$ & $\begin{array}{c}\text { Wall time } \\
(\mathrm{min})\end{array}$ \\
\hline MAVRIC-MoI & 1 & 1440 & 1440 \\
\hline MAVRIC-Shift & 256 & $1,490,010$ & 589 \\
\hline
\end{tabular}

a Total CPU time by all processors.

b Total execution time. 


\subsubsection{INDEPENDENT SPENT FUEL STORAGE INSTALLATIONS}

An alternative to permanent disposal of SNF is the Independent Spent Fuel Storage Installation (ISFSI) in which SNF storage casks are stored above ground and on site while awaiting further disposal. A simplified model of an ISFSI has been investigated by Peplow et al. [17] and is used in this report for verification purposes. The model consists of 8 casks placed on a concrete pad on top of soil surrounded by air, as shown in Fig. 28. The casks are all identical to each other and contain a homogenized fresh fuel mixture representing fuel assemblies rather than SNF for simplicity of the model. Source definitions for each cask include neutron and gamma sources which were generated via ORIGEN for typical SNF from a PWR with a discharge burnup of around 58,000 MWd/MTU and a cooling time of 10 years. The objective of the ISFSI model is to calculate the gamma dose rate around the cask and identify a boundary region where the dose is lower than the allowed limit, which is $25 \mathrm{mrem} / \mathrm{yr}$ ( or $\approx 2.85 \times 10^{-6} \mathrm{rem} / \mathrm{hr}$ ), by regulations.

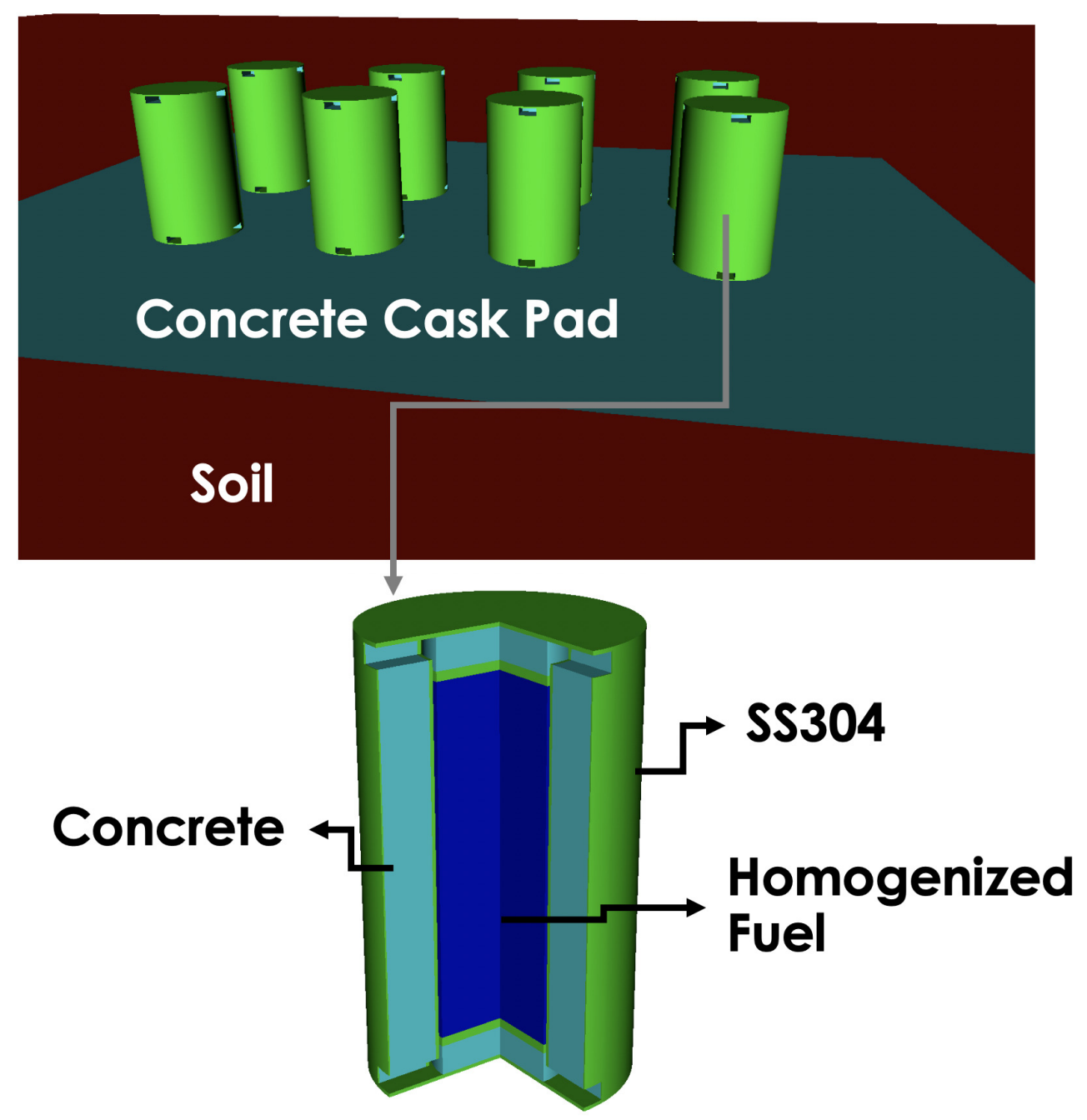

Figure 28. Simplified ISFSI model. 
A mesh tally with the response of the ANSI standard (1977) photon flux-to-dose-rate factors was used with the FW-CADIS method to optimize gamma dose rates in the air and above the ground of the cask pad. Axial and radial gamma dose rate maps at the center plane of the front row of casks from the simulations are shown in Fig. 29 and Fig. 30. Dose rates from MAVRIC-Monaco and MAVRIC-Shift are in good agreement with each other indicating that the boundary for the regulated limit is at least $1 \mathrm{~km}$ away from the cask pad. The MAVRIC-Shift results show slightly higher uncertainties for the same number of particle histories. Timing results for the simulations are given in Table 12, demonstrating substantially improved turn-around time with parallel processing in the MAVRIC-Shift simulation.

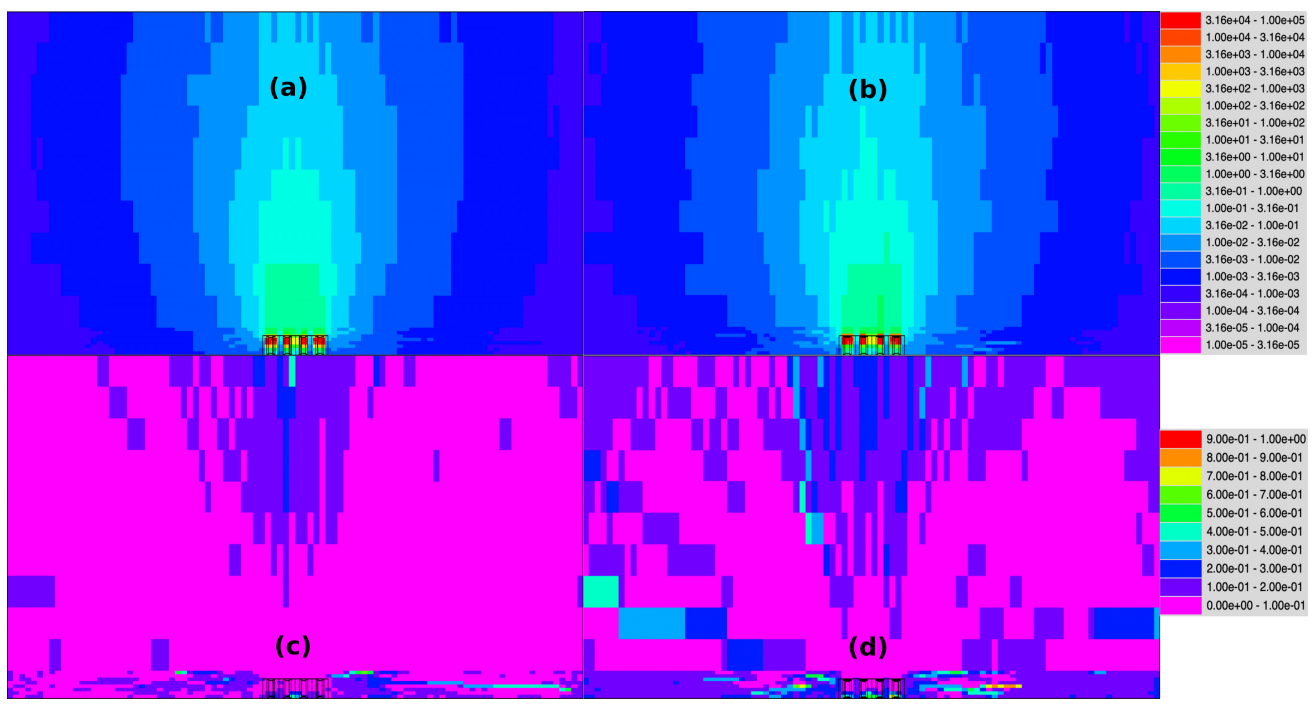

Figure 29. Axial gamma dose rate map (in rem/h) for the ISFSI model with MAVRIC-Monaco v6.3b6 (a) and MAVRIC-Shift v6.3b6 (b) with their relative uncertainties (c) and (d).

Table 12. Timing results for the ISFSI simulations.

\begin{tabular}{|c|c|c|c|}
\hline Code & $\begin{array}{c}\text { Number of } \\
\text { processors }\end{array}$ & $\begin{array}{c}\text { CPU time } \\
(\mathrm{min})\end{array}$ & $\begin{array}{c}\text { Wall time }^{\mathrm{b}} \\
(\mathrm{min})\end{array}$ \\
\hline MA & 1 & 807 & 807 \\
\hline MAVRIC-Shift [v6.3b6] & 256 & 3584 & 14 \\
\hline
\end{tabular}

a Total CPU time by all processors.

b Total execution time. 


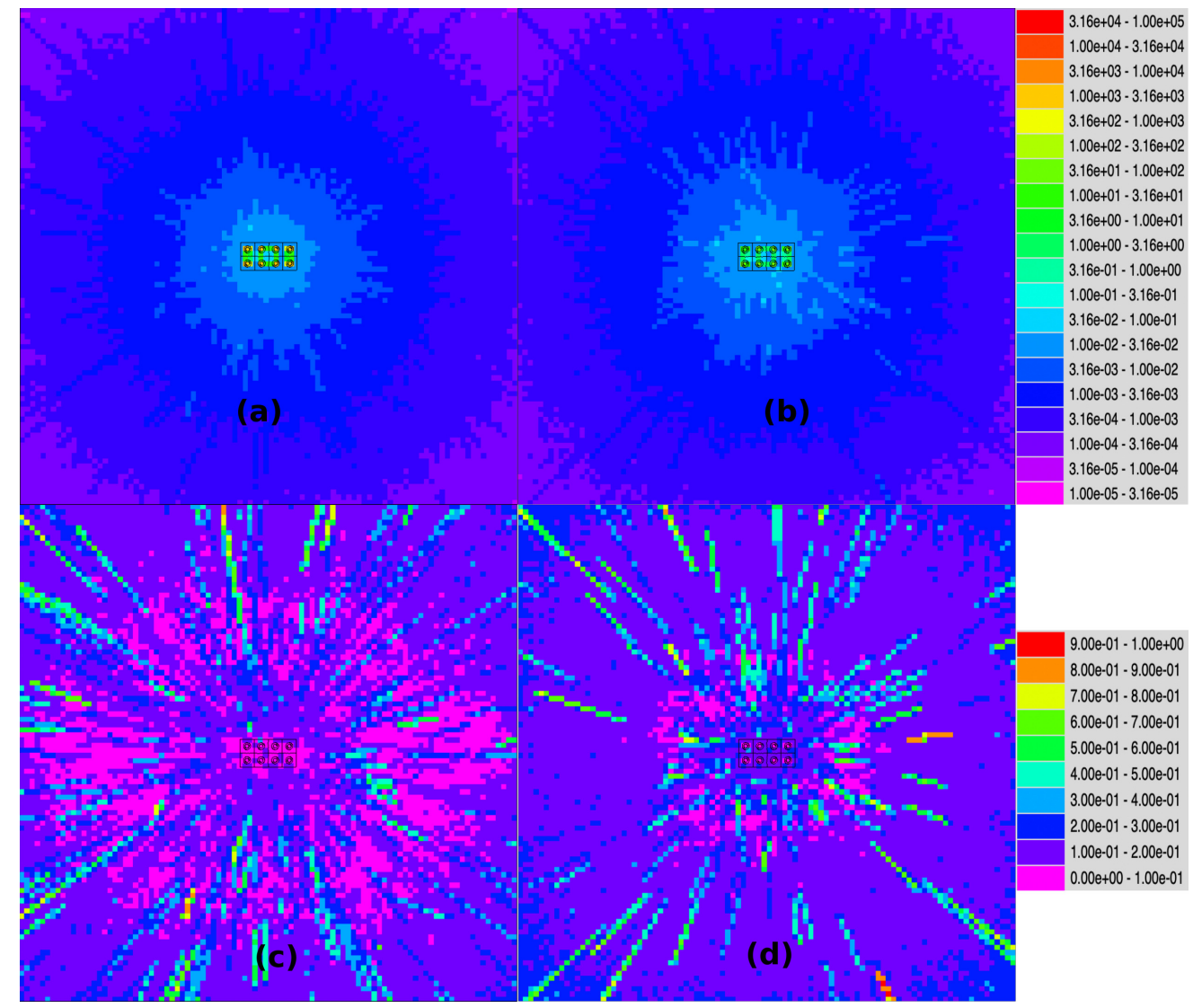

Figure 30. Radial gamma dose rate map (in rem/h) for the ISFSI model with MAVRIC-Monaco v6.3b6 (a) and MAVRIC-Shift v6.3b6 (b) with their relative uncertainties (c) and (d). 


\subsection{ADDITIONAL SHIFT VERIFICATION AND VALIDATION}

Aside from this report, other researchers have also published validation of the Shift code used by MAVRICShift to perform particle transport simulations. A short list of published research on the Shift validation is listed below.

- A preliminary validation with shielding, scattering, streaming, and skyshine simulations by Peplow et al. [18],

- Detector response function generation by Celik et al. [19],

- Neutron field measurements in a concrete labyrinth by Wagner et al. [20],

- Initial verification and validation by Pandya et al. [21],

- Implementation details and verification via benchmarks by Pandya et al. [1]. 


\section{CONCLUSIONS AND FUTURE WORK}

Selected features of the MAVRIC sequence of SCALE have been successfully implemented in the MAVRICShift sequence and integrated into the SCALE Code System, providing parallel computing capabilities for radiation transport and shielding calculations. Assessment of the MC methods with and without CADIS and FW-CADIS yielded good agreement between MAVRIC and MAVRIC-Shift. MAVRIC-Shift is ready to be used as a production-level simulation tool for shielding analysis and introduces parallel processing for faster simulations as well as more accurate representation of source terms for variance reduction methods.

Several useful features are not yet implemented into the MAVRIC-Shift sequence as development continues beyond the current beta implementation towards a production release with SCALE 6.3. A partial list of features to be supported in future releases is given below.

CAAS support

MAVRIC-Shift does not currently allow a user to generate a fission source distribution with an external eigenvalue calculation and import it as a mesh source into a shielding calculation. This feature is used in criticality accident alarm system (CAAS) simulations as well as reactor dosimetry analysis. This source specification capability is expected to be enabled in the near future.

\section{Material, region and unit filter in adjoint source}

MAVRIC-Shift does not yet have the ability to filter particle sources based on the material, region, or unit from which the particles are omitted. This is currently available in the legacy MAVRIC sequence and will be added to MAVRIC-Shift in the future.

\section{Library of flux-to-dose conversion factors}

Currently, Shift supports only histogram and linearly interpolated response functions. In the future, a complete set of response functions will be available. Storing commonly used response functions in a data library would allow users to quickly and easily apply dose responses to their simulations.

\section{Batch statistics and checks}

Shift currently only supports history-based tallies and does not feature any statistical checks; it only calculates the tally variances. In the future, Shift will support batch statistics and will provide a complete suite of statistical checks, similar to those available in Monaco.

\section{Improved output edits}

Shift does not currently output its discretized source; nor does it output the biased source. Having the biased and discretized sources available would enable users to investigate whether the source was being accurately discretized and whether the biased source behaves as expected. This capability will be added in the future.

Improve API with Fulcrum

The MAVRIC-Shift sequence archives its results in HDF5 files, a flexible, extensible, high-performance binary file format. In the future, Fulcrum will be extended to interactively interpret and visualize the data in these files.

\section{Add filtering to tallies}

The current version of Shift does not support filtering tallies based on properties of the particle or the particle source. Adding this capability would enable users to only tally particles emitted from certain materials, or from certain regions, or tally particles based on other characteristics. These features will be enabled in future releases. 
Insert unit/region/material information in 3DMap files

Currently the 3DMap files generated by MAVRIC-Shift do not have unit, region, or material information. This may be added in the future, or the 3DMap files may be deprecated in favor of a flexible, high-performance HDF5 file that could be visualized with Fulcrum.

Restart from HDF5 file

Currently, the MAVRIC-Shift sequences do not have a restart capability that would enable users to continue a previously completed calculation to obtain improved results with additional particle histories. All of the information necessary for restart is available in the current HDF5 files, but the capability to read that information and restart the simulation is not currently implemented. The capability is expected in future releases. 


\section{ACKNOWLEDGMENTS}

This work was sponsored by the US Nuclear Regulatory Commission (NRC) Office Nuclear Regulatory Research under Interagency Agreement (IAA) NRCHQ6014D0005 Task Order NRCHQ6017T0021 as well as the NRC Office of Nuclear Materials Safety and Safeguards under IAA 31310018N0004. The support of NRC program managers Don Algama and Drew Barto is gratefully acknowledged. 


\section{REFERENCES}

[1] Tara M. Pandya, Seth R. Johnson, Thomas M. Evans, Gregory G. Davidson, Steven P. Hamilton, and Andrew T. Godfrey. Implementation, capabilities, and benchmarking of Shift, a massively parallel Monte Carlo radiation transport code. Journal of Computational Physics, 308:239-272, March 2016.

[2] Bradley. T. Rearden and Matthew. A. Jessee. SCALE Code System. Technical Report ORNL/TM2005/439, Oak Ridge National Laboratory, Oak Ridge, TN, 2018.

[3] Thomas M. Evans, Alissa S. Stafford, Rachel N. Slaybaugh, and Kevin T. Clarno. Denovo: A new three-dimensional Parallel Discrete Ordinates Code in SCALE. Nuclear Technology, 171:171, 2010.

[4] Kaushik Banerjee, Kevin R. Robb, Georgeta Radulescu, John M. Scaglione, John C. Wagner, Justin B. Clarity, Robert A. Lefebvre, and Joshua L. Peterson. Estimation of inherent safety margins in loaded commercial spent nuclear fuel casks. Nuclear Technology, 195:124-142, 2016.

[5] Thomas E. Booth. A Sample Problem for Variance Reduction in MCNP. Technical Report LA-10363MS, Los Alamos National Laboratory, 1985.

[6] John C. Wagner and Alireza Haghighat. Automated variance reduction of Monte Carlo shielding calculations using the discrete ordinates adjoint function. Nuclear Science and Engineering, 128(2):186208, 1998.

[7] John C. Wagner, Scott W. Mosher, Thomas M. Evans, Douglas E. Peplow, and John A. Turner. Hybrid and parallel domain-decomposition methods development to enable Monte Carlo for reactor analyses. Progress in nuclear science and technology, 2(1):815-820, 2011.

[8] John Wagner, Douglas Peplow, and Scott Mosher. FW-CADIS Method For Global And Regional Variance Reduction Of Monte Carlo Radiation Transport Calculations. Nuclear Science and Engineering, 176(1), January 2014.

[9] S. W. Mosher, A. M. Bevill, S. R. Johnson, A. M. Ibrahim, C. R. Daily, T. M. Evans, J. C. Wagner, J. O. Johnson, and R. E. Grove. ADVANTG-An Automated Variance Reduction Parameter Generator. Technical Report ORNL/TM-2013/416, Oak Ridge National Laboratory, Oak Ridge, TN, November 2013.

[10] Kang Seog Kim, Mark L. Williams, Andrew M. Holcomb, Dorothea Wiarda, Byoung Kyu Jeon, and Won Sik Yang. The scale/ampx multigroup cross section processing for fast reactor analysis. Annals of Nuclear Energy, 132:161 - 171, 2019.

[11] E. Sajo, M.L. Williams, and M. Asgari. Comparison of measured and calculated neutron transmission through steel for a ${ }^{252} \mathrm{Cf}$ source. Annals of Nuclear Energy, 20(9):585 - 604, 1993.

[12] K. Ueki, A. Ohashi, and Y. Anayama. Neutron shielding ability of krafton n2-mannan-krafton n2 sandwich-type material and others. pages 130 - 137. ANS, ANS, April 26-May 11992.

[13] M. E. Battat, D. Harris, J. Hubbell, and R. Roussin. American national standard neutron and gammaray flux-to-dose rate factors. ANSI/ANS, 1977.

[14] Alpha-Omega Services, Inc., 2019.

[15] Dorothea Wiarda, Michael E Dunn, Douglas E Peplow, Thomas M Miller, and Hatice Akkurt. Development and testing of ENDF/B-VI. 8 and ENDF/B-VII.0 coupled neutron-gamma libraries for scale 6. NUREG Report NUREG/CR-6990, ORNL/TM-2008/047, U.S. Nuclear Regulatory Commission, Office of Nuclear Material Safety and Safeguards, Washington, DC, 2009. 
[16] Holtec International, 2019.

[17] Douglas E Peplow, Thomas M Evans, and John C Wagner. Simultaneous optimization of tallies in difficult shielding problems. Nuclear Technology, 168(3):785-792, 2009.

[18] D. E. Peplow, K. Banerjee, G. G. Davidson, I. R. Stewart, M. W. Swinney, and J. N. Wagner. Preliminary validation of the shift monte carlo code for fixed-source radiation transport problems. Nuclear Technology, 0(0):1-19, 2019.

[19] Cihangir Celik, Douglas E. Peplow, Gregory G. Davidson, and Mathew W. Swinney. A directional detector response function for anisotropic detectors. Nuclear Science and Engineering, 0(0):1-16, 2019.

[20] J. Wagner and Douglas E. Peplow. Validation of the shift monte carlo codeâĂŞneutron field measurements in a concrete labyrinth. ANS, ANS, June 9-June 132019.

[21] Tara M. Pandya, Thomas M. Evans, Gregory G Davidson, Seth R. Johnson, and Andrew T. Godfrey. Shift verification and validation. Technical Report ORNL/SR-2016/516, Oak Ridge National Laboratory, Oak Ridge, TN, 2016. 